\title{
An existence theorem for steady Navier-Stokes equations in the axially symmetric case
}

\author{
Mikhail Korobkov, Konstantin Pileckas and Remigio Russo
}

\begin{abstract}
We study the nonhomogeneous boundary value problem for the Navier-Stokes equations of steady motion of a viscous incompressible fluid in a bounded three-dimensional domain with multiply connected boundary. We prove that this problem has a solution in some axially symmetric cases, in particular, when all components of the boundary intersect the axis of symmetry.
\end{abstract}

Mathematics Subject Classification (2010): 35Q30 (primary); 76D03, 76D05 (secondary).

\section{Introduction}

Let $\Omega$ be a bounded domain in $\mathbb{R}^{3}$ with Lipschitz boundary $\partial \Omega=\Gamma_{0} \cup \ldots \cup \Gamma_{N}$, consisting of $N+1$ disjoint connected components $\Gamma_{j}$. Consider the stationary Navier-Stokes system with nonhomogeneous boundary conditions

$$
\left\{\begin{aligned}
-v \Delta \mathbf{u}+(\mathbf{u} \cdot \nabla) \mathbf{u}+\nabla p & =0 & & \text { in } \Omega, \\
\operatorname{div} \mathbf{u} & =0 & & \text { in } \Omega, \\
\mathbf{u} & =\mathbf{a} & & \text { on } \partial \Omega
\end{aligned}\right.
$$

The continuity equation $\left(1.1_{2}\right)$ implies the compatibility condition

$$
\int_{\partial \Omega} \mathbf{a} \cdot \mathbf{n} d S=\sum_{j=0}^{N} \int_{\Gamma_{j}} \mathbf{a} \cdot \mathbf{n} d S=\sum_{j=0}^{N} \mathcal{F}_{i}=0
$$

The research of M. Korobkov was supported by the Russian Foundation for Basic Research (project No. 12-01-00390-a) and by the Research Council of Lithuania (grant No. VIZIT-2-TYR005).

The research of K. Pileckas was funded by grant No. MIP-030/2011 of the Research Council of Lithuania.

The research of R. Russo was supported by the "Gruppo Nazionale per la Fisica Matematica" of "Istituto Nazionale di Alta Matematica".

Received April 2, 2012; accepted in revised form January 17, 2013. 
necessary for the solvability of (1.1), where $\mathbf{n}$ is the unit outward normal vector to $\partial \Omega$ and $\mathcal{F}_{j}=\int_{\Gamma_{j}} \mathbf{a} \cdot \mathbf{n} d S$.

Starting from the famous paper of J. Leray [23] published in 1933, problem (1.1) was studied in many articles (see, e.g., [1], [2], [8]- [13], [18]- [21], [26][36], etc.). However, for a long time the existence of a weak solution $\mathbf{u} \in W^{1,2}(\Omega)$ to problem (1.1) was established only under the assumption that

$$
\mathcal{F}_{j}=\int_{\Gamma_{j}} \mathbf{a} \cdot \mathbf{n} d S=0, \quad j=1,2, \ldots, N,
$$

or for sufficiently small fluxes (see [23], [20]- [21], [9], [36], [18], etc.). Condition (1.3) requires the flux of the boundary value a to vanish separately through each component $\Gamma_{j}$ of the boundary $\partial \Omega$, while the compatibility condition (1.2) means only that the total flux vanishes. Thus, (1.3) is stronger than (1.2) (condition (1.3) excludes the presence of sinks and sources).

A detailed survey of available results appeared in the recent papers [15] and [28]- [29]. In particular, in the latter papers Pukhnachev established the existence of a solution to (1.1) in the three-dimensional case when the domain $\Omega$ and the boundary value a have a symmetry axis and a symmetry plane perpendicular to this axis, moreover, this plane intersects each boundary component (for a more precise formulation, see below).

In this paper we study the problem in the axially symmetric case. Take coordinate axes $O_{x_{1}}, O_{x_{2}}, O_{x_{3}}$ in $\mathbb{R}^{3}$ and consider cylindrical coordinates $\theta=\operatorname{arctg}\left(x_{2} / x_{1}\right)$, $r=\left(x_{1}^{2}+x_{2}^{2}\right)^{1 / 2}, z=x_{3}$. Denote by $v_{\theta}, v_{r}, v_{z}$ the projections of a vector $\mathbf{v}$ on the axis $\theta, r, z$.

A function $f$ is said to be axially symmetric if it is independent of $\theta$. A vectorvalued function $\mathbf{h}=\left(h_{\theta}, h_{r}, h_{z}\right)$ is called axially symmetric if $h_{\theta}, h_{r}$ and $h_{z}$ are independent of $\theta$. A vector-valued function $\mathbf{h}=\left(h_{\theta}, h_{r}, h_{z}\right)$ is called axially symmetric without rotation if $h_{\theta}=0$ while $h_{r}$ and $h_{z}$ are independent of $\theta$.

We will use the following symmetry assumptions.

(SO) $\Omega \subset \mathbb{R}^{3}$ is a bounded domain with Lipschitz boundary and $O_{x_{3}}$ is a symmetry axis of $\Omega$.

(AS) The assumptions (SO) are fulfilled and the boundary value $\mathbf{a} \in W^{1 / 2,2}(\partial \Omega)$ is axially symmetric.

(ASwR) The assumptions (SO) are fulfilled and the boundary value $\mathbf{a} \in W^{1 / 2,2}(\partial \Omega)$ is axially symmetric without rotation.

Denote by $\Omega_{j}$ the bounded simply connected domain with $\partial \Omega_{j}=\Gamma_{j}, j=$ $0, \ldots, N$. Let $\Omega_{0}$ be the largest domain, i.e.,

$$
\Omega=\Omega_{0} \backslash\left(\cup_{j=1}^{N} \bar{\Omega}_{j}\right)
$$

Here and henceforth we denote by $\bar{A}$ the closure of a set $A$. 
Let

$$
\begin{aligned}
& \Gamma_{j} \cap O_{x_{3}} \neq \emptyset, \quad j=0, \ldots, M, \\
& \Gamma_{j} \cap O_{x_{3}}=\emptyset, \quad j=M+1, \ldots, N .
\end{aligned}
$$

We shall prove the existence theorem provided that one of the following two additional conditions is fulfilled:

$$
M=N-1, \quad \mathcal{F}_{N} \geq 0,
$$

or

$$
\left|\mathcal{F}_{j}\right|<\delta, \quad j=M+1, \ldots, N,
$$

where $\delta=\delta(\nu, \Omega)$ is sufficiently small (we specify $\delta(v, \Omega)$ in Section 4). In particular, (1.5) includes the case $N=M$ when each component of the boundary intersects the axis of symmetry. Notice that in (1.4), (1.5) the fluxes $\mathcal{F}_{j}, j=1, \ldots, M$, could be arbitrarily large.

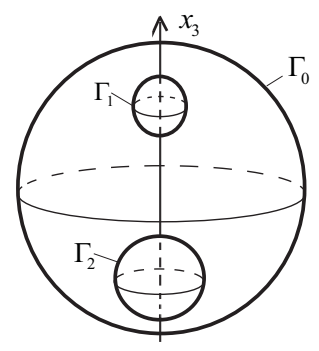

(a) $M=N=2$

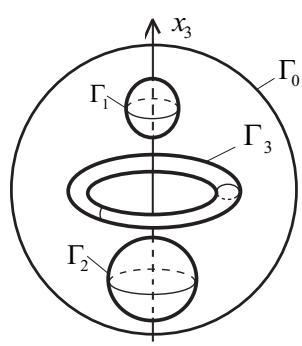

(b) $M=2, N=3$

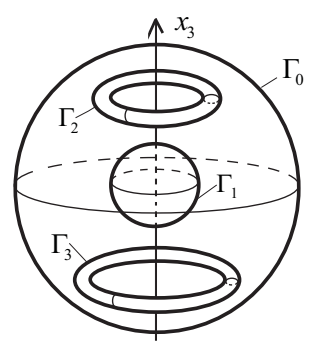

Figure 1.1. Domain $\Omega$.

(c) $M=1, N=3$

Figure 1.1 depicts several possible domains $\Omega$. In case (a) all fluxes $\mathcal{F}_{0}, \mathcal{F}_{1}$ and $\mathcal{F}_{2}$ are arbitrary; in case (b) the fluxes $\mathcal{F}_{0}, \mathcal{F}_{1}, \mathcal{F}_{2}$ are arbitrary, while $\mathcal{F}_{3}$ has to be nonnegative, but there is no restriction on its size; in the case (c) the fluxes $\mathcal{F}_{0}, \mathcal{F}_{1}$ are arbitrary, while $\mathcal{F}_{2}$ and $\mathcal{F}_{3}$ have to be "sufficiently small".

The main result of this paper reads as follows. 
Theorem 1.1. Let conditions (AS) and (1.2) be fulfilled. Suppose that either (1.4) or (1.5) holds. Then problem (1.1) admits at least one weak axially symmetric solution $\mathbf{u} \in W^{1,2}(\Omega)$.

If, in addition, conditions (ASwR) are fulfilled, then problem (1.1) admits at least one weak axially symmetric solution without rotation.

For the definition of a weak solution, see Section 2.1. Analogous results in the plane case were established in [15].

Note that in [29] the existence theorem was obtained under the following assumptions on the axially symmetric boundary data $\mathbf{a}=\left(a_{\theta}, a_{r}, a_{z}\right): a_{\theta} \equiv 0$ (i.e., the axially symmetric case without rotation), $\{z=0\}$ is a symmetry plane of $\Omega$, and each boundary component $\Gamma_{j}$ intersects this plane; furthermore, $a_{r}$ is an even function of $z$, while $a_{z}$ is an odd function of $z$ (no restrictions on the size of the fluxes). Under these assumptions, the number $N$ of boundary components can be arbitrarily large, but only at most two of them can intersect the symmetry axis, in our notation that means $M \leq 1$. Therefore, neither our Theorem 1.1 implies Pukhnachev's result, nor the latter implies the former, and so these results are in a sense independent. Moreover, the proof in [29] is based on different ideas; in particular, in [29] a priori estimates for the velocity field were obtained without using Leray's contradiction argument.

Let us also remark that Alekseev and Pukhnachev recently obtained [30] an existence theorem for the steady Navier-Stokes equations in the axially symmetric case with boundary conditions formulated in terms of stream function and vorticity. Of course, this result is even farther from our Theorem 1.1 than the previous one.

Our proof of Theorem 1.1 uses Bernoulli's law for a weak solution of the Euler equations and the weak one-sided maximum principle for the total head pressure corresponding to this solution (see Section 3). These results were obtained in [14] for the plane case (see [15] for more detailed proofs). The proof of Bernoulli's law for solutions in Sobolev spaces is based on the recent results of [3] (see also Section 2.2).

A short version of this paper appeared as [16].

ACKNOWLEDGEMEnTS. The authors are much indebted to V.V. Pukhnachev for valuable discussions.

\section{Notation and preliminary results}

By a domain we mean a connected open set. Let $\Omega \subset \mathbb{R}^{3}$ be a bounded domain with Lipschitz boundary $\partial \Omega$. We use standard notation for function spaces: $C^{k}(\bar{\Omega}), C^{k}(\partial \Omega), W^{k, q}(\Omega), \stackrel{\circ}{W}^{k, q}(\Omega), W^{\alpha, q}(\partial \Omega)$, where $\alpha \in(0,1), k \in \mathbb{N}_{0}$, and $q \in[1,+\infty]$. In our notation we do not distinguish function spaces of scalar- and vector-valued functions; it is clear from the context whether we use scalar, vector, or 
tensor-valued function spaces. Denote by $H(\Omega)$ the subspace of all solenoidal vector fields ( $\operatorname{div} \mathbf{u}=0$ ) from $\stackrel{\circ}{W}^{1,2}(\Omega)$ equipped with the norm $\|\mathbf{u}\|_{H(\Omega)}=\|\nabla \mathbf{u}\|_{L^{2}(\Omega)}$. Observe that for functions $\mathbf{u} \in H(\Omega)$ the norm $\|\cdot\|_{H(\Omega)}$ is equivalent to $\|\cdot\|_{W^{1,2}(\Omega)}$.

Working with Sobolev functions, we always assume that the "best representatives" are chosen. For $w \in L_{\text {loc }}^{1}(\Omega)$ the best representative $w^{*}$ is defined as

$$
w^{*}(x)= \begin{cases}\lim _{r \rightarrow 0} f_{B_{r}(x)} w(z) d z, & \text { if the finite limit exists } \\ 0 & \text { otherwise }\end{cases}
$$

where

$$
f_{B_{r}(x)} w(z) d z=\frac{1}{\operatorname{meas}\left(B_{r}(x)\right)} \int_{B_{r}(x)} w(z) d z,
$$

and $B_{r}(x)=\{y:|y-x|<r\}$ is the ball of radius $r$ centered at $x$.

Below (see Theorem 3.7) we discuss some properties of the best representatives for Sobolev functions.

\subsection{Some facts about solenoidal functions}

The following lemmas concern the existence of solenoidal extensions of boundary values and an integral representation for bounded linear functionals vanishing on the solenoidal functions.

Lemma 2.1 (see Corollary 2.3 in [22]). Let $\Omega \subset \mathbb{R}^{3}$ be a bounded domain with Lipschitz boundary. If $\mathbf{a} \in W^{1 / 2,2}(\partial \Omega)$ and (1.2) holds, then there exists a solenoidal extension $\mathbf{A} \in W^{1,2}(\Omega)$ of $\mathbf{a}$ with

$$
\|\mathbf{A}\|_{W^{1,2}(\Omega)} \leq c\|\mathbf{a}\|_{W^{1 / 2,2}(\partial \Omega)} .
$$

From this lemma we can deduce some assertions for the symmetric case.

Lemma 2.2. If conditions (AS) and (1.2) are fulfilled, then there exists an axially symmetric solenoidal extension $\mathbf{A} \in W^{1,2}(\Omega)$ of $\mathbf{a}$ such that estimate (2.1) holds.

Proof. Take a solenoidal extension $\mathbf{A}_{\mathbf{0}} \in W^{1,2}(\Omega)$ of a from Lemma 2.1. Put

$$
\mathbf{A}_{i}(\theta, r, z)=\frac{1}{i !} \sum_{j=0}^{i !} \mathbf{A}_{0}\left(\theta+\frac{2 \pi j}{i !}, r, z\right) .
$$

Clearly, each $\mathbf{A}_{i}$ is also a solenoidal extension of $\mathbf{a}$ and the estimate (2.1) holds for $\mathbf{A}_{i}$ with the same $c$ (independent of $i$ ). By construction

$$
\mathbf{A}_{i}\left(\theta+\frac{2 \pi j}{m}, r, z\right)=\mathbf{A}_{i}(\theta, r, z) \quad \text { for all } m=1, \ldots, i .
$$

Take a weakly convergent sequence $\mathbf{A}_{i_{k}} \rightarrow \mathbf{A}$ in $W^{1,2}(\Omega)$. Then by construction $\operatorname{div} \mathbf{A}=0,\left.\mathbf{A}\right|_{\partial \Omega}=\mathbf{a}$, and (2.1) holds. Now (2.2) implies that $\mathbf{A}\left(\theta+\frac{2 \pi j}{m}, r, z\right)=$ $\mathbf{A}(\theta, r, z)$ for all $m, j$. Hence $\mathbf{A}$ is axially symmetric. 
Lemma 2.3. Let conditions (ASwR) and (1.2) be fulfilled. Then there exists a solenoidal extension $\mathbf{A} \in W^{1,2}(\Omega)$ of $\mathbf{a}$ such that $\mathbf{A}$ is axially symmetric without rotation and estimate (2.1) holds.

Proof. Take a solenoidal extension $\tilde{\mathbf{A}}=\left(\tilde{A}_{\theta}, \tilde{A}_{r}, \tilde{A}_{z}\right) \in W^{1,2}(\Omega)$ of a from Lemma 2.2. Then a classical formula yields

$$
\begin{aligned}
\operatorname{div} \tilde{\mathbf{A}}(\theta, r, z) & =\frac{1}{r} \frac{\partial}{\partial \theta}\left(\tilde{A}_{\theta}\right)+\frac{1}{r} \frac{\partial}{\partial r}\left(\tilde{A}_{r} r\right)+\frac{\partial}{\partial z}\left(\tilde{A}_{z}\right) \\
& =\frac{1}{r} \frac{\partial}{\partial r}\left(\tilde{A}_{r} r\right)+\frac{\partial}{\partial z}\left(\tilde{A}_{z}\right)=0 .
\end{aligned}
$$

Here $\frac{\partial \tilde{A}_{\theta}}{\partial \theta}=0$ because of axial symmetry. Define the vector field $\mathbf{A}=\left(A_{\theta}, A_{r}, A_{z}\right)$ by putting

$$
A_{\theta}=0, \quad A_{r}=\tilde{A}_{r}, \quad A_{z}=\tilde{A}_{z} .
$$

Then by construction $\mathbf{A}$ is axially symmetric without rotation, $\left.\mathbf{A}\right|_{\partial \Omega}=\mathbf{a}$, and estimate (2.1) holds. Now (2.3) implies that $\operatorname{div} \mathbf{A}=0$.

Lemma 2.4 (see [33]). Let $\Omega \subset \mathbb{R}^{3}$ be a bounded domain with Lipschitz boundary and $R(\eta)$ a continuous linear functional defined on $\stackrel{\circ}{W}^{1,2}(\Omega)$. If

$$
R(\eta)=0 \quad \forall \eta \in H(\Omega),
$$

then there exists a unique function $p \in L^{2}(\Omega)$ with $\int_{\Omega} p(x) d x=0$ such that

$$
R(\boldsymbol{\eta})=\int_{\Omega} p \operatorname{div} \eta d x \quad \forall \boldsymbol{\eta} \in \stackrel{\circ}{W}^{1,2}(\Omega) .
$$

Moreover, $\|p\|_{L^{2}(\Omega)}$ is equivalent to $\|R\|_{\left(\dot{W}^{1,2}(\Omega)\right)^{*}}$.

Lemma 2.5. If, in addition to the hypotheses of Lemma 2.4, the domain $\Omega$ satisfies assumption (SO) and $R(\boldsymbol{\eta}) \equiv R\left(\boldsymbol{\eta}_{\theta_{0}}\right)$ for all $\boldsymbol{\eta} \in H(\Omega)$ and $\theta_{0} \in[0,2 \pi]$, where $\boldsymbol{\eta}_{\theta_{0}}(\theta, r, z):=\boldsymbol{\eta}\left(\theta+\theta_{0}, r, z\right)$, then the function $p$ is axially symmetric.

Proof. Take the function $p$ of Lemma 2.4. For $\theta_{0} \in[0,2 \pi]$ define a function $p_{\theta_{0}}$ by $p_{\theta_{0}}(\theta, r, z)=p\left(\theta-\theta_{0}, r, z\right)$. By construction,

$$
\begin{aligned}
\int_{\Omega} p \operatorname{div} \boldsymbol{\eta} d x & =R(\boldsymbol{\eta})=R\left(\boldsymbol{\eta}_{\theta_{0}}\right)=\int_{\Omega} p \operatorname{div} \boldsymbol{\eta}_{\theta_{0}} d x \\
& =\int_{\Omega} p_{\theta_{0}} \operatorname{div} \boldsymbol{\eta} d x \quad \forall \boldsymbol{\eta} \in \stackrel{\circ}{W}^{1,2}(\Omega) .
\end{aligned}
$$

Since $p$ is unique, the identity $p(x) \equiv p_{\theta_{0}}(x)$ follows. 
Lemma 2.6 (see [21]). Let $\Omega \subset \mathbb{R}^{3}$ be a bounded domain with Lipschitz boundary and let $\mathbf{A} \in W^{1,2}(\Omega)$ be divergence-free. Then there exists a unique weak solution $\mathbf{U} \in W^{1,2}(\Omega)$ to the Stokes problem satisfying the boundary condition $\left.\mathbf{U}\right|_{\partial \Omega}=$ $\left.\mathbf{A}\right|_{\partial \Omega}$, i.e., $\mathbf{U}-\mathbf{A} \in H(\Omega)$ and

$$
\int_{\Omega} \nabla \mathbf{U} \cdot \nabla \eta d x=0 \quad \forall \eta \in H(\Omega) .
$$

Moreover,

$$
\|\mathbf{U}\|_{W^{1,2}(\Omega)} \leq c\|\mathbf{A}\|_{W^{1,2}(\Omega)} .
$$

Lemma 2.7. If, in addition to the hypotheses of Lemma 2.6, the domain $\Omega$ satisfies assumptions ( $\mathrm{SO}$ ) and also $\mathbf{A}$ is axially symmetric, then $\mathbf{U}$ is axially symmetric too.

Proof. Take a solution $\mathbf{U}$ to the Stokes problem of Lemma 2.6. For $\theta_{0} \in[0,2 \pi] \mathrm{de}-$ fine the function $\mathbf{U}_{\theta_{0}}$ by the formula $\mathbf{U}_{\theta_{0}}(\theta, r, z):=\mathbf{U}\left(\theta-\theta_{0}, r, z\right)$. By construction, $\mathbf{U}_{\theta_{0}}-\mathbf{A} \in H(\Omega)$. Moreover,

$$
\int_{\Omega} \nabla \mathbf{U}_{\theta_{0}} \cdot \nabla \boldsymbol{\eta} d x=\int_{\Omega} \nabla \mathbf{U} \cdot \nabla \boldsymbol{\eta}_{\theta_{0}} d x=0 \quad \forall \boldsymbol{\eta} \in H(\Omega),
$$

where $\boldsymbol{\eta}_{\theta_{0}}(\theta, r, z):=\boldsymbol{\eta}\left(\theta+\theta_{0}, r, z\right)$. By uniqueness, the identity $\mathbf{U}(x) \equiv \mathbf{U}_{\theta_{0}}(x)$ follows.

Lemma 2.8. If, in addition to the hypotheses of Lemma 2.6, the vector field $\mathbf{A}$ is axially symmetric without rotation, then $\mathbf{U}$ is also axially symmetric without rotation.

Proof. Take the axially symmetric function $\mathbf{U}=\left(U_{\theta}, U_{r}, U_{z}\right)$ of Lemmas 2.6-2.7 and define $\boldsymbol{\eta}=\left(\eta_{\theta}, \eta_{r}, \eta_{z}\right)$ by putting

$$
\eta_{\theta} \equiv U_{\theta}, \quad \eta_{r}=\eta_{z} \equiv 0 .
$$

Then Lemma 2.7 implies that $\eta \in H(\Omega)$ (see also (2.3)). Consequently, (2.4) yields

$$
\int_{\Omega} \nabla \mathbf{U} \cdot \nabla \boldsymbol{\eta} d x=0 .
$$

However,

$$
\nabla \mathbf{U} \cdot \nabla \boldsymbol{\eta} \equiv\left(\frac{U_{\theta}}{r}\right)^{2}+\left(\frac{\partial U_{\theta}}{\partial r}\right)^{2}+\left(\frac{\partial U_{\theta}}{\partial z}\right)^{2}
$$

by a straightforward calculation, and the required equality $U_{\theta} \equiv 0$ follows from (2.6), (2.7). 
Given a function $\mathbf{f} \in L^{q}(\Omega)$ with $1 \leq q \leq 6 / 5$ consider the continuous linear functional $H(\Omega) \ni \eta \mapsto \int_{\Omega} \mathbf{f} \cdot \boldsymbol{\eta} d x$. By the Riesz representation theorem, there exists a unique function $\mathbf{g} \in H(\Omega)$ with

$$
\int_{\Omega} \mathbf{f} \cdot \boldsymbol{\eta} d x=\int_{\Omega} \nabla \boldsymbol{\eta} \cdot \nabla \mathbf{g} d x=\langle\mathbf{g}, \boldsymbol{\eta}\rangle_{H(\Omega)} \quad \forall \boldsymbol{\eta} \in H(\Omega) .
$$

Put $\mathbf{g}=T_{0}$ f. Evidently, $T_{0}$ is a continuous linear operator from $L^{q}(\Omega)$ to $H(\Omega)$.

Denote by $L_{A S}^{q}(\Omega)$ the space of all axially symmetric vector-valued functions in $L^{q}(\Omega)$. Similarly define the spaces $L_{A S w R}^{q}(\Omega), H_{A S}(\Omega), H_{A S w R}(\Omega), W_{A S}^{1,2}(\Omega)$, $W_{A S w R}^{1,2}(\Omega)$, etc.

Lemma 2.9. The operator $T_{0}: L^{3 / 2}(\Omega) \rightarrow H(\Omega)$ has the following symmetry properties:

$$
\begin{array}{cl}
\forall \mathbf{f} \in L_{A S}^{3 / 2}(\Omega) & T_{0} \mathbf{f} \in H_{A S}(\Omega), \\
\forall \mathbf{f} \in L_{A S w R}^{3 / 2}(\Omega) & T_{0} \mathbf{f} \in H_{A S w R}(\Omega) .
\end{array}
$$

Proof. We can prove (2.8) in the same way as Lemma 2.7 and (2.9) as Lemma 2.8 .

Lemma 2.10. The following inclusions are valid:

$$
\begin{aligned}
\forall \mathbf{u}, \mathbf{v} \in H_{A S}(\Omega) & (\mathbf{u} \cdot \nabla) \mathbf{v} \in L_{A S}^{3 / 2}(\Omega), \\
\forall \mathbf{u}, \mathbf{v} \in H_{A S w R}(\Omega) & (\mathbf{u} \cdot \nabla) \mathbf{v} \in L_{A S w R}^{3 / 2}(\Omega) .
\end{aligned}
$$

Proof. Direct calculation.

Take $\mathbf{a} \in W^{1 / 2,2}(\partial \Omega)$ and assume that conditions (1.2) and (AS) (or (ASwR)) are fulfilled. Take the corresponding axially symmetric functions $\mathbf{A}$ and $\mathbf{U}$ of Lemmas 2.2-2.3, 2.7-2.8. Put $\mathbf{w}=\mathbf{u}-\mathbf{U}$. Then problem (1.1) is equivalent to

$$
\begin{cases}-v \Delta \mathbf{w}+(\mathbf{U} \cdot \nabla) \mathbf{w}+(\mathbf{w} \cdot \nabla) \mathbf{w}+(\mathbf{w} \cdot \nabla) \mathbf{U} & \\ \quad=-\nabla p-(\mathbf{U} \cdot \nabla) \mathbf{U} & \text { in } \Omega, \\ \operatorname{div} \mathbf{w}=0 & \text { in } \Omega, \\ \mathbf{w}=0 & \text { on } \partial \Omega .\end{cases}
$$

By a weak solution to problem (1.1) we understand a function $\mathbf{u}$ such that $\mathbf{w}=$ $\mathbf{u}-\mathbf{U} \in H(\Omega)$ and

$$
\begin{aligned}
\nu\langle\mathbf{w}, \boldsymbol{\eta}\rangle_{H(\Omega)}= & -\int_{\Omega}(\mathbf{U} \cdot \nabla) \mathbf{U} \cdot \boldsymbol{\eta} d x-\int_{\Omega}(\mathbf{U} \cdot \nabla) \mathbf{w} \cdot \boldsymbol{\eta} d x \\
& -\int_{\Omega}(\mathbf{w} \cdot \nabla) \mathbf{w} \cdot \boldsymbol{\eta} d x-\int_{\Omega}(\mathbf{w} \cdot \nabla) \mathbf{U} \cdot \boldsymbol{\eta} d x \quad \forall \boldsymbol{\eta} \in H(\Omega) .
\end{aligned}
$$


By the Riesz representation theorem, for any $\mathbf{w} \in H(\Omega)$ there exists a unique function $T \mathbf{w} \in H(\Omega)$ such that the right-hand side of (2.13) is equivalent to $\langle T \mathbf{w}, \boldsymbol{\eta}\rangle_{H(\Omega)}$ for all $\eta \in H(\Omega)$. Obviously, $T$ is a nonlinear operator from $H(\Omega)$ to $H(\Omega)$.

Lemma 2.11. The operator $T: H(\Omega) \rightarrow H(\Omega)$ is compact. Moreover, $T$ has the following symmetry properties:

$$
\begin{array}{cl}
\forall \mathbf{w} \in H_{A S}(\Omega) \quad T \mathbf{w} \in H_{A S}(\Omega), \\
\forall \mathbf{w} \in H_{A S w R}(\Omega) \quad T \mathbf{w} \in H_{A S w R}(\Omega) .
\end{array}
$$

Proof. The first claim is well-known (see [21]). The symmetry claims follow from Lemmas 2.9-2.10.

Obviously, (2.13) is equivalent to the operator equation

$$
\nu \mathbf{w}=T \mathbf{w}
$$

in the space $H(\Omega)$. Thus, we can apply the Leray-Schauder fixed point theorem to the compact operators $\left.T\right|_{H_{A S}(\Omega)}$ and $\left.T\right|_{H_{A S W R}(\Omega)}$. The following statements hold.

Lemma 2.12. Let conditions (AS), (1.2) be fulfilled. Suppose that all possible solutions to the equation $\nu \mathbf{w}=\lambda T \mathbf{w}$ with $\lambda \in[0,1]$ and $\mathbf{w} \in H_{A S}(\Omega)$ are uniformly bounded in $H_{A S}(\Omega)$. Then problem (1.1) admits at least one weak axially symmetric solution.

Lemma 2.13. Let conditions (ASwR), (1.2) be fulfilled. Suppose that all possible solutions to the equation $\nu \mathbf{w}=\lambda T \mathbf{w}$ with $\lambda \in[0,1]$ and $\mathbf{w} \in H_{A S w R}(\Omega)$ are uniformly bounded in $H_{A S w R}(\Omega)$. Then problem (1.1) admits at least one weak axially symmetric solution without rotation.

\subsection{On the Morse-Sard and Luzin N-properties of Sobolev functions in $W^{2,1}$}

First we recall some classical differentiability properties of Sobolev functions.

Lemma 2.14 (see Proposition 1 in [6]). If $\psi \in W^{2,1}\left(\mathbb{R}^{2}\right)$, then $\psi$ is continuous and there exists a set $A_{\psi}$ such that $\mathfrak{H}^{1}\left(A_{\psi}\right)=0$ and $\psi$ is differentiable (in the classical sense) at each $x \in \mathbb{R}^{2} \backslash A_{\psi}$. Furthermore, the classical derivative at these points $x$ coincides with $\nabla \psi(x)=\lim _{r \rightarrow 0} f_{B_{r}(x)} \nabla \psi(z) d z$, where $\lim _{r \rightarrow 0} f_{B_{r}(x)} \mid \nabla \psi(z)-$ $\left.\nabla \psi(x)\right|^{2} d z=0$.

Here and henceforth we denote by $\mathfrak{H}^{1}$ the one-dimensional Hausdorff measure, i.e., $\mathfrak{H}^{1}(F)=\lim _{t \rightarrow 0+} \mathfrak{H}_{t}^{1}(F)$, where

$$
\mathfrak{H}_{t}^{1}(F)=\inf \left\{\sum_{i=1}^{\infty} \operatorname{diam} F_{i}: \operatorname{diam} F_{i} \leq t, F \subset \bigcup_{i=1}^{\infty} F_{i}\right\} .
$$

The following theorems have been proved recently by J. Bourgain, M. Korobkov and J. Kristensen [3]. 
Theorem 2.15. Let $\mathcal{D} \subset \mathbb{R}^{2}$ be a bounded domain with Lipschitz boundary and $\psi \in W^{2,1}(\mathcal{D})$. Then:

(i) $\mathfrak{H}^{1}\left(\left\{\psi(x): x \in \overline{\mathcal{D}} \backslash A_{\psi} \& \nabla \psi(x)=0\right\}\right)=0$;

(ii) for every $\varepsilon>0$ there exists $\delta>0$ such that for every set $U \subset \overline{\mathcal{D}}$ with $\mathfrak{H}_{\infty}^{1}(U)<\delta$ the inequality $\mathfrak{H}^{1}(\psi(U))<\varepsilon$ holds;

(iii) for $\mathfrak{H}^{1}$-almost all $y \in \psi(\overline{\mathcal{D}}) \subset \mathbb{R}$ the preimage $\psi^{-1}(y)$ is a finite disjoint family of $C^{1}$-curves $S_{j}, j=1,2, \ldots, N(y)$. Each $S_{j}$ is either a cycle in $\mathcal{D}$ (i.e., $S_{j} \subset \mathcal{D}$ is homeomorphic to the unit circle $\mathbb{S}^{1}$ ) or a simple arc with endpoints on $\partial \mathcal{D}$ (in this case $S_{j}$ is transversal to $\partial \mathcal{D}$ ).

Theorem 2.16. Let $\mathcal{D} \subset \mathbb{R}^{2}$ be a bounded domain with Lipschitz boundary and $\psi \in W^{2,1}(\mathcal{D})$. Then for every $\varepsilon>0$ there exist an open set $V \subset \mathbb{R}$ and a function $g \in C^{1}\left(\mathbb{R}^{2}\right)$ such that $\psi\left(A_{\psi}\right) \subset V, \mathfrak{H}^{1}(V)<\varepsilon$, and the identities $\psi(x) \equiv g(x)$, $\nabla \psi(x)=\nabla g(x) \neq 0$ hold for all $x \in \overline{\mathcal{D}}$ provided that $\psi(x) \notin V$.

We say that a value $y \in \psi(\overline{\mathcal{D}})$ is regular if it satisfies condition (iii) of Theorem 2.15 and $\psi(x) \notin V$ for some $g$ and $V$ of Theorem 2.16. Observe that by Theorems 2.15 and 2.16 almost all values $y \in \psi(\overline{\mathcal{D}})$ are regular.

\section{Euler equation}

We study the Euler equation under the following assumptions.

(E) Let conditions (SO) be fulfilled. Suppose that some axially symmetric functions $\mathbf{v} \in W^{1,2}(\Omega)$ and $p \in W^{1,3 / 2}(\Omega)$ satisfy the Euler system

$$
\left\{\begin{array}{l}
\lambda_{0}(\mathbf{v} \cdot \nabla) \mathbf{v}+\nabla p=0 \\
\operatorname{div} \mathbf{v}=0
\end{array}\right.
$$

for almost all $x \in \Omega$. Moreover, suppose that

$$
\left.\mathbf{v}\right|_{\partial \Omega}=0
$$

Put $P_{+}=\left\{\left(0, x_{2}, x_{3}\right): x_{2}>0, x_{3} \in \mathbb{R}\right\}, \mathcal{D}=\Omega \cap P_{+}, \mathcal{D}_{j}=\Omega_{j} \cap P_{+}$. Of course, on $P_{+}$the coordinates $x_{2}$ and $x_{3}$ coincides with $r$ and $z$. From (SO) we can easily infer that

$\left(\mathrm{S}_{1}\right) \mathcal{D}$ is a bounded plane domain with Lipschitz boundary. Moreover, $C_{j}:=$ $P_{+} \cap \Gamma_{j}$ is a connected set for each $j=0, \ldots, N$. In other words, $\left\{C_{j}: j=\right.$ $0, \ldots, N\}$ coincides with the set of all connected components of $P_{+} \cap \partial \mathcal{D}$. 
Then $\mathbf{v}$ and $p$ satisfy the following system of equations in the plane domain $\mathcal{D}$ :

$$
\left\{\begin{array}{l}
\frac{\partial p}{\partial z}+\lambda_{0} v_{r} \frac{\partial v_{z}}{\partial r}+\lambda_{0} v_{z} \frac{\partial v_{z}}{\partial z}=0 \\
\frac{\partial p}{\partial r}-\lambda_{0} \frac{\left(v_{\theta}\right)^{2}}{r}+\lambda_{0} v_{r} \frac{\partial v_{r}}{\partial r}+\lambda_{0} v_{z} \frac{\partial v_{r}}{\partial z}=0 \\
\frac{v_{\theta} v_{r}}{r}+v_{r} \frac{\partial v_{\theta}}{\partial r}+v_{z} \frac{\partial v_{\theta}}{\partial z}=0 \\
\frac{\partial\left(r v_{r}\right)}{\partial r}+\frac{\partial\left(r v_{z}\right)}{\partial z}=0
\end{array}\right.
$$

(the equations are satisfied for almost all $x \in \mathcal{D}$ ).

The next statement was proved in [13, Lemma 4] and [1, Theorem 2.2].

Theorem 3.1. If conditions (E) are fulfilled, then

$$
\forall j \in\{0, \ldots, N\} \exists p_{j} \in \mathbb{R}: \quad p(x) \equiv p_{j} \quad \text { for } \mathfrak{H}^{2} \text {-almost all } x \in \Gamma_{j} .
$$

In particular, by axial symmetry,

$$
p(x) \equiv p_{j} \quad \text { for } \mathfrak{H}^{1} \text {-almost all } x \in C_{j} .
$$

Lemma 3.2 (e.g., [18], [27]). Under the assumptions of Theorem 3.1, the estimate

$$
\max _{i, j=0, \ldots N}\left|p_{i}-p_{j}\right| \leq \delta_{1} \lambda_{0}\|\mathbf{v}\|_{H(\Omega)}^{2}
$$

holds, where the constant $\delta_{1}$ depends only on $\Omega$.

One of the main purposes of this section is to prove the following fact.

Theorem 3.3. Under the assumptions of Theorem 3.1, the equalities

$$
p_{0}=p_{1}=\cdots=p_{M}
$$

are fulfilled.

To prove the last theorem, we need some preparation, in particular, a version of Bernoulli's Law in the Sobolev case (see Theorem 3.4 below).

The last equality in (3.3) and (3.2) imply that there exists a stream function $\psi \in W_{\text {loc }}^{2,2}(\mathcal{D})$ with

$$
\frac{\partial \psi}{\partial r}=-r v_{z}, \quad \frac{\partial \psi}{\partial z}=r v_{r}
$$

We have the following integral estimates: $\mathbf{v} \in W_{\text {loc }}^{1,2}(\mathcal{D})$,

$$
\int_{\mathcal{D}} r|\mathbf{v}(r, z)|^{2} d r d z<\infty .
$$


Using (3.8), we can rewrite this as

$$
\int_{\mathcal{D}} \frac{|\nabla \psi(r, z)|^{2}}{r} d r d z<\infty .
$$

Fix a point $x_{*} \in \mathcal{D}$. For $\varepsilon>0$ denote by $\mathcal{D}_{\varepsilon}$ the connected component of $\mathcal{D} \cap$ $\{(r, z): r>\varepsilon\}$ containing $x_{*}$. Since

$$
\psi \in W^{2,2}\left(\mathcal{D}_{\varepsilon}\right) \quad \forall \varepsilon>0
$$

the Sobolev Embedding Theorem yields $\psi \in C\left(\overline{\mathcal{D}}_{\varepsilon}\right)$. Hence, $\psi$ is continuous at the points of $\overline{\mathcal{D}} \backslash O_{z}=\overline{\mathcal{D}} \backslash\{(0, z): z \in \mathbb{R}\}$.

Denote by $\Phi=p+\lambda_{0} \frac{|\mathbf{v}|^{2}}{2}$ the total head pressure corresponding to the solution $(\mathbf{v}, p)$. Obviously,

$$
\Phi \in W^{1,3 / 2}\left(\mathcal{D}_{\varepsilon}\right) \quad \forall \varepsilon>0 .
$$

Straightforward calculations yield the identity

$$
v_{r} \frac{\partial \Phi}{\partial r}+v_{z} \frac{\partial \Phi}{\partial z}=0
$$

for almost all $x \in \mathcal{D}$.

Theorem 3.4. Assume that conditions (E) are fulfilled (see the beginning of this section). Then there exists a set $A_{\mathbf{v}} \subset P_{+}$with $\mathfrak{H}^{1}\left(A_{\mathbf{v}}\right)=0$ such that if for a compact connected $^{1}$ set $K \subset \overline{\mathcal{D}} \backslash O_{z}$

$$
\left.\psi\right|_{K}=\text { const, }
$$

then

$$
\Phi\left(x_{1}\right)=\Phi\left(x_{2}\right) \text { for all } x_{1}, x_{2} \in K \backslash A_{\mathbf{v}} .
$$

Theorem 3.4 was obtained in the plane case in [14, Theorem 1] (see also [15] for a detailed proof).

To prove Theorem 3.4, we need some preliminaries.

Lemma 3.5. If conditions (E) are fulfilled, then

$$
p \in W_{\text {loc }}^{2,1}(\mathcal{D})
$$

Proof. Clearly, $p$ is the (unique) weak solution to the Poisson equation

$$
\begin{cases}\Delta p+\lambda_{0} \nabla \mathbf{v} \cdot \nabla \mathbf{v}^{\top}=0 & \text { in } \Omega \\ p=\tilde{p}, & \text { in } \partial \Omega,\end{cases}
$$

${ }^{1}$ We understand connectedness in the sense of general topology. 
with $\tilde{p}=\operatorname{tr}_{\mid \partial \Omega} p \in W^{1 / 3,3 / 2}(\partial \Omega)$. Put

$$
G(x)=\frac{\lambda_{0}}{4 \pi} \int_{\Omega} \frac{\left(\nabla \mathbf{v} \cdot \nabla \mathbf{v}^{\top}\right)(y)}{|x-y|} d v_{y} .
$$

By the results of [5], $\nabla \mathbf{v} \cdot \nabla \mathbf{v}^{\top}$ belongs to the Hardy space $\mathcal{H}^{1}\left(\mathbb{R}^{3}\right)$. Therefore, the Calderón-Zygmund theorem for Hardy spaces [34] yields $G \in W^{2,1}(\Omega)$. Take the trace $\bar{G} \in W^{1 / 3,3 / 2}(\partial \Omega)$ of $G$ on $\partial \Omega$ and the solution $p_{*} \in C^{\infty}(\Omega)$ to the problem

$$
\left\{\begin{array}{rlrl}
\Delta p_{*} & =0 & & \text { in } \Omega, \\
p_{*}=\tilde{p}-\bar{G} & & \text { in } \partial \Omega .
\end{array}\right.
$$

The uniqueness theorem yields

$$
p=p_{*}+G(x) \in W_{\mathrm{loc}}^{2,1}(\Omega) .
$$

From (3.16) we infer that $p_{r z} \equiv p_{z r}$ for almost all $x \in \mathcal{D}$. Denote $Z=\{x \in \mathcal{D}$ : $v_{r}(x)=v_{z}(x)=0$. By (3.3), we have

$$
\frac{\partial p}{\partial z}(x)=0, \quad \frac{\partial p}{\partial r}(x)=\lambda_{0} \frac{\left(v_{\theta}\right)^{2}}{r} \quad \text { for almost all } x \in Z,
$$

and it is easy to deduce that

$$
\frac{\partial \Phi}{\partial z}(x)=0 \quad \text { for almost all } x \in \mathcal{D} \text { such that } v_{r}(x)=v_{z}(x)=0 .
$$

Consider the stream function $\psi$. By (3.2) and (3.8) we have $\nabla \psi(x)=0$ for $\mathfrak{H}^{1}$ almost all $x \in \partial \mathcal{D} \backslash O_{z}$. Then the Morse-Sard property (see Theorem 2.15) implies that

$$
\text { for every connected set } C \subset \partial \mathcal{D} \backslash O_{z} \exists \alpha=\alpha(C) \in \mathbb{R}: \quad \psi(x) \equiv \alpha \forall x \in C .
$$

Then by $\left(\mathrm{S}_{1}\right)$ (see the beginning of Section 3 )

$$
\forall j \in\{0, \ldots, N\} \exists \xi_{j} \in \mathbb{R}: \quad \psi(x) \equiv \xi_{j} \forall x \in C_{j} .
$$

Remark 3.6. Since $\nabla \psi=0$ on $\partial D \backslash O_{z}$ (in the sense of traces), the function $\psi$ extends to the whole half-plane $P_{+}$:

$$
\psi(x):=\xi_{0}, x \in P_{+} \backslash \mathcal{D}_{0}, \quad \psi(x):=\xi_{j}, x \in P_{+} \cap \overline{\mathcal{D}}_{j}, j=1, \ldots, N .
$$

The functions $\mathbf{v}, p$ and $\Phi$ extend to $P_{+}$as

$$
\begin{aligned}
& \mathbf{v}(x)=0, \quad x \in P_{+} \backslash \mathcal{D}, \\
& p(x)=\Phi(x)= \begin{cases}p_{0}, & x \in P_{+} \backslash \mathcal{D}_{0}, \\
p_{j}, & x \in P_{+} \cap \overline{\mathcal{D}}_{j}, j=1, \ldots, N .\end{cases}
\end{aligned}
$$


The extended functions inherit the properties of the original ones. Namely, (3.3), (3.8)-(3.13), (3.19) hold with $\mathcal{D}$ and $\mathcal{D}_{\varepsilon}$ replaced by $P_{+}$and

$$
P_{\varepsilon}:=\left\{(r, z): r \in\left[\varepsilon, \frac{1}{\varepsilon}\right], z \in\left[-\frac{1}{\varepsilon}, \frac{1}{\varepsilon}\right]\right\},
$$

respectively. $z \in \mathbb{R}\}$.

For $r_{0}>0$ denote by $L_{r_{0}}$ the straight line parallel to the $z$-axis: $L_{r_{0}}=\left\{\left(r_{0}, z\right)\right.$ :

Working with Sobolev functions, we always assume that the "best representatives" are chosen. We collect the basic properties of these "best representatives" in the next theorem.

Theorem 3.7. There exists a set $A_{\mathbf{v}} \subset P_{+}$with the following properties.

(i) $\mathfrak{H}^{1}\left(A_{\mathbf{v}}\right)=0$.

(ii) For all $x \in P_{+} \backslash A_{\mathbf{v}}$

$$
\begin{aligned}
& \lim _{r \rightarrow 0} f_{B_{r}(x)}|\mathbf{v}(y)-\mathbf{v}(x)|^{2} d y=\lim _{r \rightarrow 0} f_{B_{r}(x)}|\Phi(y)-\Phi(x)|^{3 / 2} d y=0, \\
& \lim _{r \rightarrow 0} \frac{1}{r} \int_{B_{r}(x)}|\nabla \Phi(y)|^{3 / 2} d y=0,
\end{aligned}
$$

and moreover, the function $\psi$ is differentiable at $x$ and $\nabla \psi(x)=\left(-r v_{z}(x)\right.$, $\left.r v_{r}(x)\right)$.

(iii) For all $\varepsilon>0$ there exists an open set $U \subset \mathbb{R}^{2}$ with $\mathfrak{H}_{\infty}^{1}(U)<\varepsilon$ and $A_{\mathbf{v}} \subset U$ such that the functions $\mathbf{v}$ and $\Phi$ are continuous on $P_{+} \backslash U$.

(iv) For each $x_{0}=\left(r_{0}, z_{0}\right) \in P_{+} \backslash A_{\mathbf{v}}$ and for every $\varepsilon>0$ we have the convergence

$$
\lim _{\rho \rightarrow 0+} \frac{1}{2 \rho} \mathfrak{H}^{1}\left(E\left(x_{0}, \varepsilon, \rho\right)\right) \rightarrow 1,
$$

where

$$
\begin{aligned}
& E\left(x_{0}, \varepsilon, \rho\right):=\left\{t \in(-\rho, \rho): \int_{r_{0}-\rho}^{r_{0}+\rho}\left|\frac{\partial \Phi}{\partial r}\left(r, z_{0}+t\right)\right| d r+\int_{z_{0}-\rho}^{z_{0}+\rho}\left|\frac{\partial \Phi}{\partial z}\left(r_{0}+t, z\right)\right| d z\right. \\
&+\sup _{r \in\left[r_{0}-\rho, r_{0}+\rho\right]}\left|\Phi\left(r, z_{0}+t\right)-\Phi\left(x_{0}\right)\right| \\
&\left.+\sup _{z \in\left[z_{0}-\rho, z_{0}+\rho\right]}\left|\Phi\left(r_{0}+t, z\right)-\Phi\left(x_{0}\right)\right|<\varepsilon\right\} .
\end{aligned}
$$

(v) Take a function $g \in C^{1}\left(\mathbb{R}^{2}\right)$ and a closed set $F \subset P_{+}$such that $\nabla g \neq 0$ on $F$. Then for almost all $y \in g(F)$ and for all connected components $K$ of the set $F \cap g^{-1}(y)$ we have $K \cap A_{\mathbf{v}}=\emptyset$, the restriction $\left.\Phi\right|_{K}$ is an absolutely continuous function, while (3.3) and (3.13) hold $\mathfrak{H}^{1}$-almost everywhere on $K$. 
Most of these properties are from [7]. For a detailed proof of Theorem 3.7 see, for example, [15]. The property (iv) follows directly from (3.25). The last property (v) follows (by coordinate transformation, $c f$. [24, Section 1.1.7]) from the well-known fact that every function $f \in W^{1,1}$ is absolutely continuous along almost all coordinate lines. The same fact together with (3.19) and (3.13) implies

Lemma 3.8. For almost all $r_{0}>0$ we have $L_{r_{0}} \cap A_{\mathbf{v}}=\emptyset$; moreover, $p\left(r_{0}, \cdot\right)$ and $\mathbf{v}\left(r_{0}, \cdot\right)$ are absolutely continuous functions (locally) and

$$
\frac{\partial \Phi}{\partial z}\left(r_{0}, z\right)=0 \quad \text { for almost all } z \in \mathbb{R} \text { with } v_{r}\left(r_{0}, z\right)=0 .
$$

Below we prove that Bernoulli's Law (Theorem 3.4) holds with the set $A_{\mathbf{v}}$ from Theorem 3.7. This requires several lemmas.

Lemma 3.9. For almost all $y \in \psi\left(P_{+}\right)$we have

$$
\psi^{-1}(y) \cap A_{\mathbf{v}}=\emptyset,
$$

and for each continuum ${ }^{2} K \subset \psi^{-1}(y)$ the identities

$$
\Phi\left(x_{1}\right)=\Phi\left(x_{2}\right) \text { for all } x_{1}, x_{2} \in K
$$

hold.

Proof. Fix some $\varepsilon>0$ and consider a function $g \in C^{1}\left(\mathbb{R}^{2}\right)$ and an open set $V$ with $\mathfrak{H}^{1}(V)<\varepsilon$ from Theorem 2.16 applied to the function $\left.\psi\right|_{P_{\varepsilon}}$, where the rectangle $P_{\varepsilon}$ is defined by (3.24). Put $F=P_{\varepsilon} \backslash \psi^{-1}(V)$. Then $\psi(x)=g(x)$ and $\nabla \psi(x)=$ $\nabla g(x) \neq 0$ for all $x \in F$. Thus, by Theorem 3.7 (v) for almost all $y \in \psi\left(P_{\varepsilon}\right) \backslash V=$ $g(F)$ and for every connected component $K$ of the set $\left\{x \in P_{\varepsilon}: \psi(x)=y\right\}$ the equality $K \cap A_{\mathbf{v}}=\emptyset$ holds and the restriction $\left.\Phi\right|_{K}$ is absolutely continuous; moreover, for every $C^{1}$-smooth parametrization $\gamma:[0,1] \rightarrow K$ the identity (3.13) gives

$$
[\Phi(\gamma(t))]^{\prime}=\nabla \Phi(\gamma(t)) \cdot \gamma^{\prime}(t)=0 \quad \text { for } \mathfrak{H}^{1} \text {-almost all } t \in[0,1]
$$

(the last equality is valid because $\psi(x)=$ const on $K$, and hence $\nabla \psi(\gamma(t)) \cdot \gamma^{\prime}(t)=$ $\left.r\left(-v_{z}(\gamma(t)), v_{r}(\gamma(t))\right) \cdot \gamma^{\prime}(t)=0\right)$. Thus, $\Phi(x)=$ const on $K$. Since $\varepsilon>0$ is arbitrary, the assertion of the lemma follows.

We also need certain technical facts about the continuity properties of $\Phi$ at "good" points $x \in P_{+} \backslash A_{\mathbf{v}}$.

Lemma 3.10. Take $x_{0} \in P_{+} \backslash A_{\mathbf{v}}$. Suppose that there exist a constant $\sigma>0$ and a sequence of continua $K_{j} \subset P_{+} \backslash A_{\mathbf{v}}$ with $\left.\Phi\right|_{K_{j}} \equiv \beta_{j}$ and $K_{j} \subset B_{x_{0}}\left(\rho_{j}\right)$, where $\rho_{j} \rightarrow 0$ as $j \rightarrow \infty$, and $\operatorname{diam}\left(K_{j}\right) \geq \sigma \rho_{j}$. Then $\beta_{j} \rightarrow \Phi\left(x_{0}\right)$ as $j \rightarrow \infty$.

${ }^{2}$ By a continuum we mean a compact connected set. 
Proof. Without loss of generality we may assume that the projection of each $K_{j}$ on the $O_{r}$-axis is a segment $I_{j} \subset\left[r_{0}-\rho_{j}, r_{0}+\rho_{j}\right]$ of length $\geq \frac{1}{2} \sigma \rho_{j}$ (otherwise the same fact holds for the projection of $K_{j}$ on the $O_{z}$-axis). By Theorem 3.7 (iv), for every $\varepsilon>0$ we have $I_{j}-\left\{r_{0}\right\} \cap E\left(x_{0}, \varepsilon, \rho_{j}\right) \neq \varnothing$ for sufficiently large $j$. Thus, $\left|\beta_{j}-\Phi\left(x_{0}\right)\right|<\varepsilon$ for sufficiently large $j$.

Lemma 3.11. Suppose that for $r_{0}>0$ the assertion of Lemma 3.8 is fulfilled, i.e., $L_{r_{0}} \cap A_{\mathbf{v}}=\emptyset, p\left(r_{0}, \cdot\right)$ and $\mathbf{v}\left(r_{0}, \cdot\right)$ are absolutely continuous functions, and formula (3.27) is valid. Assume that $F \subset \mathbb{R}$ is a compact set such that

$$
\psi\left(r_{0}, z\right) \equiv \text { const } \text { for all } z \in F
$$

and

$$
\Phi\left(r_{0}, \alpha\right)=\Phi\left(r_{0}, \beta\right) \quad \text { for every interval }(\alpha, \beta) \text { adjoining } F
$$

(recall that $(\alpha, \beta)$ is called an interval adjoining $F$ if $\alpha, \beta \in F$ and $(\alpha, \beta) \cap F=\emptyset)$. Then

$$
\Phi\left(r_{0}, z\right) \equiv \text { const for all } z \in F
$$

Proof. Take a pair $z^{\prime}, z^{\prime \prime} \in F$ with $z^{\prime}<z^{\prime \prime}$. Define a function $g(z)$ on the interval $\left[z^{\prime}, z^{\prime \prime}\right]$ by the rule $g(z)=\Phi\left(r_{0}, z\right)$. By construction, $g(\cdot)$ is an absolutely continuous function, and (3.31) implies that $g(\alpha)=g(\beta)$ for every interval $(\alpha, \beta) \subset\left[z^{\prime}, z^{\prime \prime}\right]$ adjoining $F$. Since by definition the absolutely continuous function $g(z)$ is differentiable almost everywhere and coincides with the Lebesgue integral of its derivative, we obtain

$$
\int_{\alpha}^{\beta} g^{\prime}(z) d z=0 .
$$

Hence,

$$
\int_{\mu}^{v} g^{\prime}(z) d z=0
$$

if $\mu, v \in F \cap\left[z^{\prime}, z^{\prime \prime}\right]$ and the interval $(\mu, v)$ contains only finitely many points of $F$. Consider now the closed set

$F_{\infty}=\left\{z \in\left[z^{\prime}, z^{\prime \prime}\right]:\right.$ every neighborhood of $z$ contains infinitely many points of $\left.F\right\}$.

By (3.33),

$$
\int_{\left[z^{\prime}, z^{\prime \prime}\right] \backslash F_{\infty}} g^{\prime}(z) d z=0 .
$$


According to properties (ii) of Theorem 3.7, the function $\psi$ is differentiable at every point $\left(r_{0}, z\right)$ with $z \in\left(z^{\prime}, z^{\prime \prime}\right)$. Hence, (3.30) yields $\psi_{z}\left(r_{0}, z\right)=0$ for all $z \in F_{\infty}$. Using (3.8), we can rewrite the last fact as $v_{r}\left(r_{0}, z\right)=0$ for all $z \in F_{\infty}$. Then (3.27) immediately implies that

$$
\int_{F_{\infty}} g^{\prime}(z) d z=0 .
$$

Adding up (3.34) and (3.35), we obtain

$$
g\left(z^{\prime}\right)-g\left(z^{\prime \prime}\right)=\int_{z^{\prime}}^{z^{\prime \prime}} g^{\prime}(z) d z=0 .
$$

This is equivalent to the required equality $\Phi\left(r_{0}, z^{\prime}\right)=\Phi\left(r_{0}, z^{\prime \prime}\right)$. The proof of the lemma is complete.

\section{Proof of Theorem 3.4 .}

STEP 1. By Remark 3.6, we may assume without loss of generality that the continuum $K$ is a connected component of the set $\left\{x \in P: \psi(x)=y_{0}\right\}$, where $y_{0} \in \mathbb{R}$ and $P \subset P_{+}$is a rectangle $P:=\left\{(r, z): r \in\left[r_{1}, r_{2}\right], z \in\left[z_{1}, z_{2}\right]\right\}$ with $r_{1}>0$, while $\psi(x) \equiv \xi_{0}$ and $\Phi(x) \equiv p_{0}$ for each $x \in \partial^{*} P$, where we denote

$$
\partial^{*} P=\partial P \backslash\left\{\left(r_{1}, z\right): z \in\left(z_{1}, z_{2}\right)\right\} .
$$

Put $P^{\circ}=$ Int $P=\left(r_{1}, r_{2}\right) \times\left(z_{1}, z_{2}\right)$. Given $\varepsilon>0$, denote by $K_{\varepsilon}$ the connected component of the compact set $\left\{x \in P: \psi(x) \in\left[y_{0}-\varepsilon, y_{0}+\varepsilon\right]\right\}$ which includes $K$. Clearly, $K_{\varepsilon} \rightarrow K$ as $\varepsilon \rightarrow 0$ in the Hausdorff metric ${ }^{3}$. By Theorem 2.15 and Lemma 3.9 for almost all $\varepsilon>0$ the set $P^{\circ} \cap \partial K_{\varepsilon}$ is a finite disjoint union of $C^{1}$-curves on which the functions $\psi$ and $\Phi$ are constant. This implies that for each component $U_{i}$ of the open set $P^{\circ} \backslash K$ there exists a sequence of continua $K_{j}^{i} \subset \bar{U}_{i} \backslash A_{\mathbf{v}}$ such that each $K_{j}^{i}$ is a $C^{1}$-curve homeomorphic to the segment $[0,1]$ or to the circle $\mathbb{S}^{1}$, furthermore, $K_{j}^{i}$ is a connected component of the set $\left\{x \in P: \psi(x)=\alpha_{j}^{i} \neq y_{0}\right\},\left.\Phi\right|_{K_{j}^{i}} \equiv \beta_{j}^{i}, K_{j}^{i} \rightarrow K \cap \partial U_{i}$ as $j \rightarrow \infty$ in the Hausdorff metric, and for every $x \in U_{i}$ there exists an index $j_{x}$ such that $x$ and $K$ lie in the different connected components of the set $P \backslash K_{j}^{i}$ for $j \geq j_{x}$. Using Lemma 3.10, it is easy to deduce from these facts that for every $U_{i}$ the limit $\beta_{i}=\lim _{j \rightarrow \infty} \beta_{j}^{i}$ exists and

$$
\Phi(x)=\beta_{i} \quad \text { for all } x \in K \cap \partial U_{i} \backslash A_{\mathbf{v}} .
$$

${ }^{3}$ Recall that the Hausdorff metric $d_{H}$ between two compact sets $A, B \subset \mathbb{R}^{n}$ is defined as follows: $d_{H}(A, B)=\max \left(\sup _{a \in A} \operatorname{dist}(a, B), \sup _{b \in B} \operatorname{dist}(b, A)\right)(e . g ., \S 7.3 .1$ in [4]). 
STEP 2. We claim that for almost all $r_{0} \in\left(r_{1}, r_{2}\right)$ the identities

$$
\Phi\left(r_{0}, z^{\prime}\right)=\Phi\left(r_{0}, z^{\prime \prime}\right) \quad \forall\left(r_{0}, z^{\prime}\right),\left(r_{0}, z^{\prime \prime}\right) \in K
$$

hold. Indeed, take $r_{0} \in\left(r_{1}, r_{2}\right)$ satisfying the assertion of Lemma 3.8 and points $\left(r_{0}, z^{\prime}\right),\left(r_{0}, z^{\prime \prime}\right) \in K$. Put $F=\left\{z \in\left[z^{\prime}, z^{\prime \prime}\right]:\left(r_{0}, z\right) \in K\right\}$. By (3.36), we infer that $\Phi\left(r_{0}, \alpha\right)=\Phi\left(r_{0}, \beta\right)$ for every interval $(\alpha, \beta) \subset\left[z^{\prime}, z^{\prime \prime}\right]$ adjoining $F$. Thus, the required identity (3.37) follows directly from Lemma 3.11.

STEP 3. We claim that there exists $\beta_{0} \in \mathbb{R}$ with

$$
\beta_{i} \equiv \beta_{0}
$$

for each component $U_{i}$ (see formula (3.36)). We split the proof of this claim into two cases.

(3a) Suppose that $K \cap \partial^{*} P \neq \emptyset$. Then by construction (see the beginning of Step 1) $y_{0}=\xi_{0}, K \supset \partial^{*} P,\left.\Phi\right|_{\partial^{*} P} \equiv p_{0}$, and we easily deduce from (3.36)(3.37) that $\beta_{i} \equiv p_{0}$.

(3b) Suppose now that $K \cap \partial^{*} P=\emptyset$. Let $U_{1}$ be the component with $\partial^{*} P \subset \partial U_{1}$. Then for each horizontal line $L_{r_{0}}$ if $L_{r_{0}} \cap K \neq \emptyset$, then $L_{r_{0}} \cap K \cap \partial U_{1} \neq \emptyset$. Hence, (3.36)-(3.37) imply that $\beta_{i} \equiv \beta_{1}$. This justifies (3.38).

Now we can rewrite (3.36)-(3.37) as

$$
\begin{gathered}
\Phi(x)=\beta_{0} \quad \text { for all } x \in K \cap \partial U_{i} \backslash A_{\mathbf{v}} \text { and each } i, \\
\Phi(r, z)=\beta_{0} \quad \text { for almost all } r \in\left(r_{1}, r_{2}\right) \text { and for every }(r, z) \in K
\end{gathered}
$$

(here $\beta_{0}$ equals either $p_{0}$ or $\beta_{1}$ ).

STEP 4. We claim that

$$
\Phi\left(x_{0}\right)=\beta_{0}
$$

for every $x_{0} \in K \backslash A_{\mathbf{v}}$. Indeed, fix $x_{0}=\left(r_{0}, z_{0}\right) \in K \backslash A_{\mathbf{v}}$ (for simplicity we suppose that $\left.x_{0} \in P^{\circ}\right)$. We divide the proof of the claim into two cases.

(4a) Suppose that there exists $\delta>0$ such that $K \cap\left\{\left(r_{0}+t, z\right):\left|z-z_{0}\right| \leq|t|\right\} \neq \emptyset$ for every $t \in(-\delta, \delta)$. Then (3.41) follows from (3.40) and assertion (iv) of Theorem 3.7. Namely, fix $\varepsilon>0$ and take $t \in(-\delta, \delta) \cap E\left(x_{0}, \varepsilon, \rho\right)$ (this intersection is nonempty for sufficiently small $\rho$ ) such that $L_{r_{0}+t} \cap A_{\mathbf{v}}=\varnothing$ and (3.40) holds for $r=r_{0}+t$, i.e.,

$$
\Phi\left(r_{0}+t, z\right)=\beta_{0} \quad \text { for every } z \text { with }\left(r_{0}+t, z\right) \in K .
$$

By construction, $|t|<\rho$. By our assumption (4a) there exists a point $\left(r_{0}+\right.$ $\left.t, z_{t}\right) \in K$ with $\left|z_{t}-z_{0}\right| \leq|t|<\rho$. Theorem 3.7 (iv) implies that $\mid \Phi\left(r_{0}+\right.$ $\left.t, z_{t}\right)-\Phi\left(x_{0}\right) \mid<\varepsilon$. Using (3.42), we finally obtain $\left|\beta_{0}-\Phi\left(x_{0}\right)\right|<\varepsilon$. 
(4b) Suppose now that assumption (4a) is false. Then there exists a sequence $0 \neq t_{k} \rightarrow 0$ with

$$
K \cap\left\{\left(r_{0}+t_{k}, z\right):\left|z-z_{0}\right| \leq\left|t_{k}\right|\right\}=\emptyset .
$$

This implies that each segment $\left\{\left(r_{0}+t_{k}, z\right):\left|z-z_{0}\right| \leq\left|t_{k}\right|\right\}$ is included into some $U_{i_{k}}$. Denote by $Q_{k}$ the open squares $Q_{k}=\left(r_{0}-\left|t_{k}\right|, r_{0}+\left|t_{k}\right|\right) \times$ $\left(z_{0}-\left|t_{k}\right|, z_{0}+\left|t_{k}\right|\right)$. It is easy to deduce that for sufficiently large $k$ each set $\overline{Q_{k} \cap K \cap \partial U_{i_{k}}}$ includes a continuum $K_{k}$ with $\operatorname{diam}\left(K_{k}\right) \geq\left|t_{k}\right|$. Indeed, by construction there exists $r_{k} \in\left[r_{0}, r_{0}+t_{k}\right)$ with $\left(r_{k}, z_{0}\right) \in \partial U_{i_{k}}$ (the existence of $r_{k}$ follows from the inclusions $\left(r_{0}, z_{0}\right) \in K \subset \mathbb{R}^{2} \backslash U_{i_{k}}$ and $\left.\left(r_{0}+t_{k}, z_{0}\right) \in U_{i_{k}}\right)$. Take as $K_{k}$ the closure of the connected component of $Q_{k} \cap \partial U_{i_{k}}$ containing the point $\left(r_{k}, z_{0}\right)$. Then $K_{k} \cap \partial Q_{k} \neq \emptyset$, as otherwise there would be a contradiction with the connectedness of $K$. However, $K_{k}$ does not intersect the segment $\left\{\left(r_{0}+t_{k}, z\right):\left|z-z_{0}\right| \leq\left|t_{k}\right|\right\}$ by assumption (3.43). Hence, $K_{k}$ intersects at least one of the other three sides of $\partial Q_{k}$. In each case diam $\left(K_{k}\right) \geq\left|t_{k}\right|$. Therefore, (3.41) follows from (3.39) and Lemma 3.10.

This justifies (3.41) for all $x_{0} \in K \backslash A_{\mathbf{v}}$. Thus, the proof of Theorem 3.4 is complete.

Proof of Theorem 3.3. To prove (3.7), we use Bernoulli's law and the fact that the axis $O_{z}$ is "almost" a stream line. More precisely, $O_{z}$ is a singularity line for $\mathbf{v}$, $\psi$ and $p$, but it can be accurately approximated by regular stream lines (on which $\Phi=$ const $)$.

First of all, let us simplify the geometrical setting. Put

$$
\tilde{\mathcal{D}}=\mathcal{D} \cup \overline{\mathcal{D}}_{M+1} \cup \cdots \cup \overline{\mathcal{D}}_{N}
$$

and consider extensions of $\psi$ and $\Phi$ to $\tilde{\mathcal{D}}$ by the formulas of Remark 3.6. Then the extended functions $\psi$ and $\Phi$ inherit the properties of the original ones. Namely, Bernoulli's Law (see the assertion of Theorem 3.4) and (3.10)-(3.12) hold with $\mathcal{D}$ and $\mathcal{D}_{\varepsilon}$ replaced by $\tilde{\mathcal{D}}$ and $\tilde{\mathcal{D}}_{\varepsilon}$. Below these facts suffice. Thus, we may assume without loss of generality that $N=M$, i.e., that $\tilde{\mathcal{D}}=\mathcal{D}$ is a simply connected plane domain.

By (3.10), there exists a sequence $r_{i} \rightarrow 0+$ such that

$$
\int_{L_{i}}|\nabla \psi| d z \rightarrow 0 \quad \text { as } i \rightarrow+\infty
$$

for the lines $L_{i}=\left\{(r, z) \in \overline{\mathcal{D}}: r=r_{i}\right\}$. Fix a point $x_{0} \in \mathcal{D}$ and denote by $\mathcal{D}^{i}$ the connected component of the open set $\left\{(r, z) \in \mathcal{D}: r>r_{i}\right\}$ containing $x_{0}$. Obviously, for sufficiently large $i$ the open set $\mathcal{D}^{i}$ is a simply connected plane domain 
with a Lipschitz boundary, $\psi \in W^{2,1}\left(\mathcal{D}^{i}\right) \subset C\left(\overline{\mathcal{D}}^{i}\right)$. In addition,

$$
\begin{aligned}
\partial \mathcal{D}^{i} \backslash L_{i} & =C_{0}^{i} \cup \cdots \cup C_{M}^{i}, \\
C_{j}^{i} \cap L_{i} & \neq \emptyset, \quad j=0, \ldots, M,
\end{aligned}
$$

where $C_{j}^{i}=C_{j} \cap\left\{(r, z) \in \mathcal{D}: r \geq r_{i}\right\}, j=0, \ldots, M$. Using (3.20) and (3.45), we conclude that

$$
\operatorname{diam}\left(\psi\left(\partial \mathcal{D}^{i}\right)\right)=\sup _{x, y \in \partial \mathcal{D}^{i}}|\psi(x)-\psi(y)| \rightarrow 0 .
$$

In particular, $\xi_{0}=\cdots=\xi_{M}$, i.e.,

$$
\left.\left.\psi\right|_{P_{+} \cap \partial \mathcal{D}} \equiv \xi_{0} \equiv \psi\right|_{\partial \mathcal{D}^{i} \backslash L_{i}}, \quad \sup _{x \in \partial \mathcal{D}^{i}}\left|\psi(x)-\xi_{0}\right| \rightarrow 0 .
$$

Our plan for the remainder of the proof is as follows. First, we prove that for every $x \in P_{+} \cap \overline{\mathcal{D}}$ there exists a set $U(x)$ such that

$$
\begin{gathered}
x \in U(x) \subset P_{+} \cap \overline{\mathcal{D}}, \quad O_{z} \cap \partial U(x) \neq \emptyset, \\
\left.\psi\right|_{P_{+} \cap \partial U(x)} \equiv \xi_{0}, \\
\exists \beta(x) \in \mathbb{R}: \quad \Phi(y)=\beta(x) \forall y \in P_{+} \cap(\partial U(x)) \backslash A_{\mathbf{v}} .
\end{gathered}
$$

Observe that $\left.\psi\right|_{P_{+} \cap \partial U(x)}=\xi_{0}$ is independent of $x$, while $\left.\Phi\right|_{P_{+} \cap \partial U(x)}=\beta(x)$ can a priori depend on $x$. However, we prove eventually that $\beta(x) \equiv p_{0}$ for all $x \in P_{+} \cap \overline{\mathcal{D}}$. This fact will easily imply the required equalities (3.7).

Define an equivalence relation on $\overline{\mathcal{D}}^{i}$ by the rule $x \sim_{i} y \Leftrightarrow \exists$ a continuum $K \subset \overline{\mathcal{D}}^{i}$ such that $\left.\psi\right|_{K} \equiv$ const and both $x, y$ lie outside the unbounded connected component of the open set $\mathbb{R}^{2} \backslash K$. Denote by $U_{i}(x)$ the corresponding equivalence class. Let us illustrate this definition by some examples.

(I ) If $K \subset \overline{\mathcal{D}}^{i}$ is a continuum and $\left.\psi\right|_{K}=$ const, then $x \sim_{i} y$ for all $x, y \in K$.

(II ) If $K \subset \overline{\mathcal{D}}^{i}$ is homeomorphic to the circle and $\left.\psi\right|_{K} \equiv$ const, then $x \sim_{i} y$ for all $x, y \in U$, where $U$ is a bounded domain with $\partial U=K$.

The following properties of the relation $\sim_{i}$ hold for every $x \in \overline{\mathcal{D}}^{i}$ (for a proof see Appendix).

(III $\sim) \quad U_{i}(x) \subset U_{i+1}(x)$ and every $U_{i}(x)$ is a compact set.

$(\mathrm{IV} \sim)$ The set $U_{i}(x)$ is connected.

$\left.\left(\mathrm{V}_{\sim}\right) \quad \psi\right|_{\partial U_{i}(x)} \equiv$ const.

(VI ) The set $\mathbb{R}^{2} \backslash U_{i}(x)$ is connected.

(VII $\sim$ ) The set $\partial U_{i}(x)$ is connected. 
(VIII ) We have

$$
L_{i} \cap \partial U_{i}(x) \neq \emptyset .
$$

For $x \in \overline{\mathcal{D}} \backslash O_{z}$ put $U(x)=\bigcup_{i} U_{i}(x)$. It is topologically obvious that

$$
\forall y \in P_{+} \cap \partial U(x) \exists \text { a sequence } \partial U_{i}(x) \ni y_{i} \rightarrow y .
$$

Then ( $\left.\mathrm{V}_{\sim}\right),(3.48)-(3.49)$, and (3.52) imply (3.50).

Bernoulli's Law (see Theorem 3.4) implies that

$$
\forall x \in P_{+} \cap \overline{\mathcal{D}} \exists \beta_{i}(x): \quad \Phi(y)=\beta_{i}(x) \text { for all } y \in \partial U_{i}(x) \backslash A_{\mathbf{v}} .
$$

Fix a point $y_{*} \in P_{+} \cap \partial U(x) \backslash A_{\mathbf{v}}$ and $j$ such that $y_{*} \in \overline{\mathcal{D}}^{j} \backslash L_{j}$. By construction (see properties ( $\left.\mathrm{VII}_{\sim}\right)-\left(\mathrm{VIII}_{\sim}\right)$ and (3.53) ) there exist sequences of continua $K_{i} \subset$ $\overline{\mathcal{D}}^{j} \cap \partial U_{i}(x)$ and points $y_{i} \in K_{i}$ such that $K_{i} \cap L_{j} \neq \emptyset$ for all sufficiently large $i$, $y_{i} \rightarrow y_{*}$, and $K_{i}$ converges as $i \rightarrow \infty$ to some set $K$ with respect to the Hausdorff metric. Hence, $y_{*} \in K, K$ is a compact connected set, $\left.\psi\right|_{K} \equiv \xi_{0}=$ const, and $K \cap L_{j} \neq \emptyset$. Consequently,

$$
\operatorname{diam} K>0 .
$$

Again Bernoulli's Law implies that

$$
\exists \beta \in \mathbb{R}: \quad \Phi(y)=\beta \text { for all } y \in K \backslash A_{\mathbf{v}} .
$$

Using (3.54)-(3.55), the connectedness of $K$ and $K_{i}$, and the continuity properties of $\Phi$ (see Theorem 3.7 (iii) ), we obtain

$$
\lim _{i \rightarrow \infty} \beta_{i}(x)=\beta .
$$

In particular,

$$
\Phi\left(y_{*}\right)=\lim _{i \rightarrow \infty} \beta_{i}(x) .
$$

Because the right-hand side here is independent of the choice of $y_{*} \in P_{+} \cap \partial U(x) \backslash$ $A_{\mathbf{v}}$, we have justified (3.51) with $\beta(x)=\lim _{i \rightarrow \infty} \beta_{i}(x)$.

Now take $r_{0}>0$ satisfying the assertion of Lemma 3.8 and the points $\left(r_{0}, z^{\prime}\right)$, $\left(r_{0}, z^{\prime \prime}\right) \in P_{+} \cap \partial \mathcal{D}$ such that $\left\{\left(r_{0}, z\right): z \in\left(z^{\prime}, z^{\prime \prime}\right)\right\} \subset \mathcal{D}$. To complete the proof of the theorem, we must show that

$$
\Phi\left(r_{0}, z^{\prime}\right)=\Phi\left(r_{0}, z^{\prime \prime}\right) .
$$

Put

$$
F=\left\{z \in\left[z^{\prime}, z^{\prime \prime}\right]:\left(r_{0}, z\right) \in \partial U\left(\left(r_{0}, z\right)\right)\right\} .
$$

Then by construction $z^{\prime}, z^{\prime \prime} \in F$ and the set $F$ is compact. Indeed, put $x^{\prime}=\left(r_{0}, z^{\prime}\right)$ and $x^{\prime \prime}=\left(r_{0}, z^{\prime \prime}\right)$. Since $U\left(x^{\prime}\right) \subset P_{+} \cap \overline{\mathcal{D}}$, we have $\partial \mathcal{D} \ni x^{\prime} \notin \operatorname{Int} U\left(x^{\prime}\right)$, and consequently, $x^{\prime} \in \partial U\left(x^{\prime}\right)$. Similarly, $x^{\prime \prime} \in \partial U\left(x^{\prime \prime}\right)$, i.e., $z^{\prime}, z^{\prime \prime} \in F$. Furthermore, 
take $z_{k} \rightarrow z_{0}, z_{k} \in F$. Put $x_{k}=\left(r_{0}, z_{k}\right)$. Then $x_{k} \in \partial U\left(x_{k}\right)$ and $x_{k} \rightarrow x_{0}=$ $\left(r_{0}, z_{0}\right)$. Of course, $x_{0} \notin \operatorname{Int} U\left(x_{0}\right)$, for otherwise $x_{k} \in \operatorname{Int} U\left(x_{0}\right)=\operatorname{Int} U\left(x_{k}\right)$ for large $k$. Therefore, $x_{0} \in \partial U\left(x_{0}\right)$, i.e., $z_{0} \in F$. Hence, $z^{\prime}, z^{\prime \prime} \in F$ and $F$ is a compact set.

Now (3.50)-(3.51) yield (3.30)-(3.31). Thus, Lemma 3.11 implies the required equality (3.57).

In the course of the last proof we established in particular the following assertion.

Lemma 3.12. Assume that conditions (E) are fulfilled. Let $K_{i}$ be a sequence of compact sets such that $K_{i} \subset \overline{\mathcal{D}} \cap P_{+},\left.\psi\right|_{K_{i}}=$ const, and there exist $x_{i}, y_{i} \in K_{i}$ with $\operatorname{dist}\left(x_{i}, O_{z}\right) \rightarrow 0$ and $\operatorname{dist}\left(y_{i}, O_{z}\right) \nrightarrow 0$. Then there exist $\beta_{i} \in \mathbb{R}$ such that $\Phi(x) \equiv \beta_{i}$ for all $x \in K_{i} \backslash A_{\mathbf{v}}$ and $\beta_{i} \rightarrow p_{0}$ as $i \rightarrow \infty$.

Let $U \subset \mathbb{R}^{2}$ be a domain with Lipschitz boundary. We say that a function $f \in W^{1, s}(U)$ satisfies the weak one-sided maximum principle locally in $U$ if

$$
\underset{x \in U^{\prime}}{\operatorname{ess} \sup ^{\prime}} f(x) \leq \underset{x \in \partial U^{\prime}}{\operatorname{ess} \sup } \operatorname{f(x)}
$$

for every strictly interior subdomain $U^{\prime}\left(\right.$ i.e., $\left.\bar{U}^{\prime} \subset U\right)$ whose boundary $\partial U^{\prime}$ avoids the singleton connected components. (In (3.58) the negligible sets are those of the two-dimensional Lebesgue measure zero in the left esssup, and those of the onedimensional Hausdorff measure zero in the right esssup.)

If (3.58) holds for every $U^{\prime} \subset U$ (not necessarily strictly interior) whose boundary $\partial U^{\prime}$ avoids the singleton connected components, then we say that $f \in$ $W^{1, s}(U)$ satisfies the weak one-sided maximum principle globally in $U$ (in particular, we can take $U^{\prime}=U$ in (3.58)).

Theorem 3.13. Let conditions (E) be fulfilled. Assume that there exists a sequence of functions $\left\{\Phi_{\mu}\right\}$ such that $\Phi_{\mu} \in W_{\mathrm{loc}}^{1, s}(\mathcal{D})$ and $\Phi_{\mu} \rightarrow \Phi$ weakly in $W_{\mathrm{loc}}^{1, s}(\mathcal{D})$ for some $s \in[4 / 3,2)$. If all $\Phi_{\mu}$ satisfy the weak one-side maximum principle locally in $\mathcal{D}$, then

$$
\underset{x \in \mathcal{D}}{\operatorname{ess} \sup } \Phi(x) \leq \max _{j=0, \ldots, N} p_{j} .
$$

Proof. If the hypotheses of Theorem 3.13 hold, then Theorem 2 of [14] (see also [15] for a more detailed proof) implies

(*) for every subdomain $U \subset \mathcal{D}$ with $\bar{U} \cap O_{z}=\emptyset$ the function $\left.\Phi\right|_{\bar{U}}$ satisfies the weak one-sided maximum principle globally.

In order to prove the estimate (3.59) on the whole domain $\mathcal{D}$, we use the same methods as in the proof of Theorem 3.3. First of all, we simplify the situation: as above, define the domain $\tilde{\mathcal{D}}$ by (3.44) and extend the functions $\psi$ and $\Phi$ into $\tilde{\mathcal{D}}$ using (3.21)-(3.23). The extended functions $\psi$ and $\Phi$ inherit the properties of the original ones. Namely, (3.10)-(3.12) and Bernoulli's Law (see Theorem 3.4) hold 
with $\mathcal{D}$ and $\mathcal{D}_{\varepsilon}$ replaced by $\tilde{\mathcal{D}}$ and $\tilde{\mathcal{D}}_{\varepsilon}$. Moreover, the maximum property (*) holds with $\mathcal{D}$ replaced by $\tilde{\mathcal{D}}$. Since these facts suffice for the proof below, we may assume without loss of generality that $N=M$, i.e., $\tilde{\mathcal{D}}=\mathcal{D}$ is a simply connected plane domain.

Suppose that the assertion of the theorem is false. Then there exists a point $x_{*} \in \mathcal{D} \backslash A_{\mathbf{v}}$ with

$$
\Phi\left(x_{*}\right)=p_{*}>\max _{j=0, \ldots, N} p_{j} .
$$

Take a sequence of numbers $r_{i} \rightarrow+0$, the corresponding lines $L_{i}$ and domains $\mathcal{D}^{i}$ as in the proof of Theorem 3.3 (in particular, (3.45) holds). Denote by $K_{i}^{*}$ the connected component of the level set $\left\{x \in \overline{\mathcal{D}}^{i}: \psi(x)=\psi\left(x_{*}\right)\right\}$ containing $x_{*}$. By Bernoulli's Law,

$$
\Phi(x)=p_{*} \quad \text { for all } i \text { and for all } x \in K_{i}^{*} \backslash A_{\mathbf{v}} .
$$

There are two possibilities:

(I) $K_{i}^{*} \cap L_{i} \neq \emptyset$ for all $i$. Then Lemma 3.12 yields $p_{*}=p_{0}$ and we arrive at a contradiction with assumption (3.60).

(II) There exists $i_{0}$ with $K_{i_{0}}^{*} \cap L_{i_{0}}=\emptyset$. Then the sequence $K_{i}^{*}$ stabilizes after $i=i_{0}$ :

$$
K_{i}^{*}=K_{i_{0}}^{*}, \quad K_{i}^{*} \cap L_{i}=\emptyset \quad \text { for all } i \geq i_{0} .
$$

Put $K^{*}=K_{i_{0}}^{*}$. Then by construction

$$
K^{*} \cap \partial \mathcal{D}^{i}=\emptyset \quad \text { for all } i \geq i_{0} .
$$

Now consider the family of sets $U_{i}\left(x_{*}\right)$ introduced in the proof of Theorem 3.3. By (3.54),

$$
\Phi(y)=\beta_{i}\left(x_{*}\right) \quad \forall y \in \partial U_{i}\left(x_{*}\right) \backslash A_{\mathbf{v}},
$$

where

$$
\lim _{i \rightarrow \infty} \beta_{i}\left(x_{*}\right)=p_{0}
$$

(the last convergence follows from Lemma 3.12). Take sufficiently large $i_{1} \geq i_{0}$ with

$$
\beta_{i}\left(x_{*}\right)<p_{*} \quad \text { for all } i \geq i_{1} .
$$

Put $U=\operatorname{Int} U_{i_{1}}\left(x_{*}\right)$. By construction,

$$
\underset{x \in U}{\operatorname{ess} \sup } \Phi(x) \geq p_{*}>\beta_{i_{1}}\left(x_{*}\right)=\underset{x \in \partial U}{\operatorname{ess} \sup } \Phi(x) .
$$

However, this contradicts $(*)$. The proof of Theorem 3.13 is complete. 


\section{Proof of the existence theorem}

Consider firstly the axially symmetric case with possible rotation. According to Lemma 2.12, in order to prove the existence of a solution to problem (1.1), it suffices to show that all possible solutions to the operator equation

$$
\nu \mathbf{w}=\lambda T \mathbf{w}, \lambda \in[0,1], \mathbf{w} \in H_{A S}(\Omega)
$$

are uniformly bounded in $H_{A S}(\Omega)$. We prove this estimate by contradiction, following the famous argument of J. Leray [23] (many other authors used it, e.g., [20], [21], [13], [1], see also [15]).

Suppose that the solutions to (4.1) are not uniformly bounded in $H_{A S}(\Omega)$. Then there exists a sequence of functions $\mathbf{w}_{k} \in H_{A S}(\Omega)$ such that $\nu \mathbf{w}_{\mathbf{k}}=\lambda_{k} T \mathbf{w}_{\mathbf{k}}$ with $\lambda_{k} \in[0,1]$ and $J_{k}=\left\|\mathbf{w}_{k}\right\|_{H(\Omega)} \rightarrow \infty$. Observe that $\mathbf{w}_{k}$ and the corresponding axially symmetric pressures $p_{k} \in L_{A S}^{2}(\Omega)$ satisfy the integral identity

$$
\begin{aligned}
v \int_{\Omega} \nabla \mathbf{w}_{k} \cdot \nabla \boldsymbol{\eta} d x= & -\lambda_{k} \int_{\Omega}(\mathbf{U} \cdot \nabla) \mathbf{U} \cdot \boldsymbol{\eta} d x-\lambda_{k} \int_{\Omega}(\mathbf{U} \cdot \nabla) \mathbf{w}_{k} \cdot \boldsymbol{\eta} d x \\
& -\lambda_{k} \int_{\Omega}\left(\mathbf{w}_{k} \cdot \nabla\right) \mathbf{w}_{k} \cdot \boldsymbol{\eta} d x-\lambda_{k} \int_{\Omega}\left(\mathbf{w}_{k} \cdot \nabla\right) \mathbf{U} \cdot \boldsymbol{\eta} d x \\
& +\int_{\Omega} p_{k} \operatorname{div} \boldsymbol{\eta} d x
\end{aligned}
$$

for every $\eta \in \stackrel{\circ}{W}^{1,2}(\Omega)$. Here $\mathbf{U}$ is an axially symmetric solution to the Stokes problem (see Lemmas 2.6-2.7).

Put $\mathbf{u}_{k}=\mathbf{w}_{k}+\mathbf{U}, \widehat{\mathbf{u}}_{k}=\frac{1}{J_{k}} \mathbf{u}_{k}, \widehat{\mathbf{w}}_{k}=\frac{1}{J_{k}} \mathbf{w}_{k}$, and $\widehat{p}_{k}=\frac{1}{J_{k}^{2}} p_{k}$. Then $\left\|\widehat{\mathbf{w}}_{k}\right\|_{H(\Omega)}=1$ and we have

$$
\left\|\widehat{p}_{k}\right\|_{L^{2}(\Omega)} \leq \mathrm{const}, \quad\left\|\widehat{p}_{k}\right\|_{W^{1,3 / 2}\left(\Omega^{\prime}\right)} \leq \mathrm{const}
$$

for every $\bar{\Omega}^{\prime} \subset \Omega$ (for a detailed proof of the above estimates see [15] for instance). Extracting subsequences, we may assume without loss of generality that

$$
\begin{gathered}
\lambda_{k} \rightarrow \lambda_{0} \in[0,1], \\
\widehat{\mathbf{u}}_{k} \rightarrow \mathbf{v} \in H(\Omega) \quad \text { weakly in } W^{1,2}(\Omega), \\
\widehat{p}_{k} \rightarrow p \in W_{\mathrm{loc}}^{1,3 / 2}(\Omega) \cap L^{2}(\Omega) \quad \text { weakly in } L^{2}(\Omega) \text { and in } W_{\mathrm{loc}}^{1,3 / 2}(\Omega) .
\end{gathered}
$$

Multiplying (4.2) for an arbitrary fixed $\eta \in \stackrel{\circ}{W}^{1,2}(\Omega)$ by $J_{k}^{-2}$ and passing to the limit as $k \rightarrow \infty$, we find that the limit functions $\mathbf{v}$ and $p$ satisfy the Euler equations

$$
\left\{\begin{array}{l}
\lambda_{0}(\mathbf{v} \cdot \nabla) \mathbf{v}+\nabla p=0, \\
\operatorname{div} \mathbf{v}=0 \\
\left.\mathbf{v}\right|_{\partial \Omega}=0
\end{array}\right.
$$


(for details of the proof see [15] for example). By (4.4) and (4.5), this implies that $p \in W^{1,3 / 2}(\Omega)$. Thus, assumptions (E) in the beginning of Section 3 are fulfilled. Moreover, $\|\mathbf{v}\|_{H(\Omega)} \leq 1$.

Now, taking $\boldsymbol{\eta}=J_{k}^{-2} \mathbf{w}_{k}$ in (4.2), we obtain

$$
v \int_{\Omega}\left|\nabla \widehat{\mathbf{w}}_{k}\right|^{2} d x=\lambda_{k} \int_{\Omega}\left(\widehat{\mathbf{w}}_{k} \cdot \nabla\right) \widehat{\mathbf{w}}_{k} \cdot \mathbf{U} d x+J_{k}^{-1} \lambda_{k} \int_{\Omega}(\mathbf{U} \cdot \nabla) \widehat{\mathbf{w}}_{k} \cdot \mathbf{U} d x
$$

Using the compact embedding $H(\Omega) \hookrightarrow L^{r}(\Omega)$ with $r<6$, we can pass to the limit as $k \rightarrow \infty$ in equality (4.7). This yields

$$
\nu=\lambda_{0} \int_{\Omega}(\widehat{\mathbf{v}} \cdot \nabla) \widehat{\mathbf{v}} \cdot \mathbf{U} d x .
$$

From the last formula and the Euler equation (4.6), we derive

$$
v=-\int_{\Omega} \nabla p \cdot \mathbf{U} d x=-\int_{\Omega} \operatorname{div}(p \mathbf{U}) d x=-\int_{\partial \Omega} p \mathbf{a} \cdot \mathbf{n} d S .
$$

Because of (3.4), we can rearrange this as

$$
\sum_{j=0}^{N} p_{j} \mathcal{F}_{j}=-v
$$

Now, using (1.2) and (3.7), we deduce from (4.10) that

$$
p_{0} \sum_{j=0}^{M} \mathcal{F}_{j}+\sum_{j=M+1}^{N} p_{j} \mathcal{F}_{j}=\sum_{j=M+1}^{N} \mathcal{F}_{j}\left(p_{j}-p_{0}\right)=-v .
$$

To begin with, consider case (1.5). If condition (1.5) is fulfilled with $\delta=\frac{1}{\delta_{1}(N-M)} v$, where $\delta_{1}$ is a constant of Lemma 3.2, then (4.11) and (3.6) lead to a contradiction (recall that $\|\mathbf{v}\|_{H(\Omega)} \leq 1$ and $\lambda_{0} \in[0,1]$ ). Thus, the proof of case (1.5) is complete.

Consider now the case that condition (1.4) is fulfilled. Then (4.11) becomes

$$
\mathcal{F}_{N}\left(p_{0}-p_{N}\right)=v \text {. }
$$

By (1.4) and (4.12),

$$
p_{0}>p_{N}
$$

Consider the identity

$$
\begin{aligned}
\operatorname{div}\left(x p+\lambda_{0}(\mathbf{v} \cdot x) \mathbf{v}\right) & =\left(x \cdot \nabla p+x \cdot \lambda_{0}(\mathbf{v} \cdot \nabla) \mathbf{v}\right)+3 p+\lambda_{0}|\mathbf{v}|^{2} \\
& =3\left(p+\frac{\lambda_{0}}{2}|\mathbf{v}|^{2}\right)-\frac{\lambda_{0}}{2}|\mathbf{v}|^{2}=3 \Phi-\frac{\lambda_{0}}{2}|\mathbf{v}|^{2} .
\end{aligned}
$$


Integrating by parts in $\Omega$, we obtain

$$
\begin{aligned}
3 \int_{\Omega} \Phi d x-\frac{\lambda_{0}}{2} \int_{\Omega}|\mathbf{v}|^{2} d x & =\int_{\partial \Omega} p(x \cdot \mathbf{n}) d S \\
& =p_{0} \int_{\Gamma_{0}}(x \cdot \mathbf{n}) d S+p_{0} \sum_{j=1}^{N-1} \int_{\Gamma_{j}}(x \cdot \mathbf{n}) d S+p_{N} \int_{\Gamma_{N}}(x \cdot \mathbf{n}) d S \\
& =p_{0} \int_{\Omega_{0}} \operatorname{div} x d x-p_{0} \sum_{j=1}^{N-1} \int_{\Omega_{j}} \operatorname{div} x d x-p_{N} \int_{\Omega_{N}} \operatorname{div} x d x \\
& =3 p_{0}\left(\left|\Omega_{0}\right|-\sum_{j=1}^{N-1}\left|\Omega_{j}\right|\right)-3 p_{N}\left|\Omega_{N}\right| \\
& =3 p_{0}|\Omega|+3\left(p_{0}-p_{N}\right)\left|\Omega_{N}\right| .
\end{aligned}
$$

Hence,

$$
\int_{\Omega} \Phi d x \geq \int_{\Omega} \Phi d x-\frac{\lambda_{0}}{6} \int_{\Omega}|\mathbf{v}|^{2} d x=p_{0}|\Omega|+\left(p_{0}-p_{N}\right)\left|\Omega_{N}\right| .
$$

The total head pressures $\Phi_{k}=p_{k}+\frac{\lambda_{k}}{2}\left|\mathbf{u}_{k}\right|^{2}$ for the Navier-Stokes system (1.1) satisfy the equations

$$
v \Delta \Phi_{k}-\lambda_{k} \mathbf{u}_{k} \cdot \nabla \Phi_{k}=v\left|\operatorname{curl} \mathbf{u}_{k}\right|^{2} \geq 0
$$

Hence (e.g., [25]), $\Phi_{k}$ satisfy the one-sided maximum principle locally in $\Omega$. Put $\widehat{\Phi}_{k}=\frac{1}{J_{k}^{2}} \Phi_{k}$. By (4.4)-(4.5) and the symmetry assumptions, the sequence $\left\{\widehat{\Phi}_{k}\right\}$ weakly converges to $\Phi=p+\frac{\lambda_{0}}{2}|\mathbf{v}|^{2}$ in the space $W_{\text {loc }}^{1,3 / 2}(\mathcal{D})$. Therefore, by Theorem 3.13,

$$
\underset{x \in \Omega}{\operatorname{ess} \sup } \Phi(x)=\underset{x \in \mathcal{D}}{\operatorname{ess} \sup } \Phi(x) \leq \max _{j=0, \ldots, N} p_{j}=p_{0}
$$

(the last equality follows from the conditions $N=M+1$ and (4.13)). Then (4.14) yields

$$
p_{0}|\Omega|+\left(p_{0}-p_{N}\right)\left|\Omega_{N}\right| \leq p_{0}|\Omega| \quad \Leftrightarrow \quad p_{0} \leq p_{N},
$$

and we arrived at a contradiction with (4.13), which proves the theorem in case (1.4).

If the boundary value a is axially symmetric without rotation, then the proof of Theorem 1.1 is the same as in the first part; we only have to use Lemma 2.13 instead of Lemma 2.12 . 


\section{Appendix}

Let us establish the topological properties (III $\sim)$-(VIII $\sim$ ) of the equivalence class $U_{i}(x)$ for $x \in \overline{\mathcal{D}}^{i}$ used in the proof of Theorem 3.3.

(III ) Indeed, if $U_{i}(x) \ni y_{j} \rightarrow y$, then by definition there exists a sequence of continua $K_{j}$ with $\left.\psi\right|_{K_{j}}=$ const such that $x$ and $y_{j}$ lie outside the unbounded connected component of the set $\mathbb{R}^{2} \backslash K_{j}$. Without loss of generality we may assume that $K_{j}$ converge in the Hausdorff metric to a set $K$. Then $K$ is a continuum, $\left.\psi\right|_{K}=$ const, and it is easy to see that neither $x$ nor $y$ belongs to the unbounded connected component of the open set $\mathbb{R}^{2} \backslash K$.

(IV ) Fix some $y \in U_{i}(x)$. Take the corresponding set $K$ in the definition of $x \sim_{i}$ $y$. Then $K \subset \overline{\mathcal{D}}^{i}$ is a compact connected set with $\left.\psi\right|_{K} \equiv$ const such that both $x$ and $y$ lie outside the unbounded connected component of the open set $\mathbb{R}^{2} \backslash K$. Denote the family of connected components of $\mathbb{R}^{2} \backslash K$ by $V_{j}$ and take an unbounded component $V_{0}$. Since $\mathcal{D}^{i}$ is a simply connected domain, we have $\bar{V}_{j} \subset \overline{\mathcal{D}}^{i}$ for all $j \neq 0$. Hence, the definition of $\sim_{i}$ yields $\bar{V}_{j} \subset U_{i}(x)$ for all $j \neq 0$. By construction, the sets $K$ and $\bar{V}_{j}$ are connected and $K \cap \bar{V}_{j} \neq \emptyset$. Therefore, the set $S_{y}=K \cup\left(\bigcup_{j \neq 0} \bar{V}_{j}\right)$ is connected and $\{x, y\} \subset S_{y} \subset U_{i}(x)$. Since $y \in U_{i}(x)$ is arbitrary, the connectedness of $U_{i}(x)$ follows.

( $\mathrm{V} \sim)_{\sim}$ To prove that $\left.\psi\right|_{\partial U_{i}(x)}=$ const, we may assume without loss of generality that $x \in \partial U_{i}(x)$. Fix some $y \in \partial U_{i}(x)$. Take the corresponding set $K$ in the definition of $x \sim_{i} y$ and the sets $V_{j}$ in the proof of property (IV ). Then it is easy to see that

$$
x, y \in K \text {. }
$$

Indeed, if $y \notin K$ for example, then $y \in V_{j}$ for some $j \neq 0$. But by construction $V_{j}$ is an open set and $V_{j} \subset U_{i}(x)$, in contradiction with the assumption that $y \in$ $\partial U_{i}(x)$. This proves (5.1), using which and the assumption $\left.\psi\right|_{K} \equiv$ const, we obtain the required equality $\psi(y)=\psi(x)$.

Using similar elementary arguments, we can easily prove the next two properties $\left(\mathrm{VI}_{\sim}\right)-\left(\mathrm{VII}_{\sim}\right)$. Therefore, we prove in detail only the last property $\left(\mathrm{VIII}_{\sim}\right)$.

(VIII ) Suppose that (3.52) fails, i.e.,

$$
L_{i} \cap \partial U_{i}(x)=\emptyset .
$$

Hence,

$$
L_{i} \cap U_{i}(x)=\emptyset .
$$

By $\left(\mathrm{V}_{\sim}\right),\left.\psi\right|_{\partial U_{i}(x)}$ equals some constant, denote it by $c_{0}$. Fix $y_{0} \in \partial U_{i}(x)$. Properties $\left(\mathrm{I}_{\sim}\right),\left(\mathrm{V}_{\sim}\right)$ and $\left(\mathrm{VII}_{\sim}\right)$ yield

$$
\partial U_{i}(x) \subset K_{0} \subset U_{i}(x),
$$


where we denote by $K_{0}$ the connected component of the level set $\left\{y \in \overline{\mathcal{D}}^{i}: \psi(y)=\right.$ $c_{0}$ \} containing the point $y_{0}$.

By construction, the closure of each connected component $\tilde{C}$ of $\left(\partial \mathcal{D}^{i}\right) \backslash L_{i}$ intersects the line $L_{i}$ and $\left.\psi\right|_{\tilde{C}} \equiv$ const (see (3.46)-(3.47), (3.49)). Hence, conditions (5.3)-(5.4) imply that

$$
K_{0} \cap \partial \mathcal{D}^{i}=U_{i}(x) \cap \partial \mathcal{D}^{i}=\emptyset .
$$

Take a sequence $0<\delta_{j} \rightarrow 0$ such that all values $c_{0}+\delta_{j}$ and $c_{0}-\delta_{j}$ are regular from the viewpoint of the Morse-Sard theorem (see Theorem 2.15 (iii)). Denote by $B_{j}$ the connected component of the level set $\left\{y \in \overline{\mathcal{D}}^{i}: \psi(y) \in\left[c_{0}-\delta_{j}, c_{0}+\delta_{j}\right]\right\}$ containing $K_{0}$. Then for sufficiently large $j$ the boundary $\partial B_{j}$ amounts to a finite disjoint family of $C^{1}$-cycles in $\mathcal{D}^{i}$ (this follows from (5.5) and from the evident convergence $\left.\sup \operatorname{dist}\left(y, K_{0}\right) \rightarrow 0\right)$.

$$
y \in B_{j}
$$

Denote by $K_{j} \subset \partial B_{j}$ the cycle separating $B_{j}$ from infinity, and by $U_{j}$ the bounded domain with $\partial U_{j}=K_{j}$. Then by construction $\left.\psi\right|_{K_{j}} \equiv$ const, $K_{0} \cap K_{j}=$ $\emptyset$, and $K_{0} \subset U_{j}$. Consequently,

$$
U_{i}(x) \varsubsetneqq U_{j}
$$

On the other hand, by property (II $\sim$ ) all points of $U_{j}$ are $\sim_{i}$ equivalent, which contradicts (5.6) and the definition of $U_{i}(x)$. This justifies (3.52).

ADDED IN PROOF. After the paper was submitted, these results and techniques allowed us to prove the existence theorem for plane and 3D axially symmetric spatial stationary flows in the general situation: under the necessary and sufficient condition of the zero total flux (see [17]).

\section{References}

[1] CH. J. AMICK, Existence of solutions to the nonhomogeneous steady Navier-Stokes equations, Indiana Univ. Math. J. 33 (1984), 817-830.

[2] W. BorCHERS and K. PILECKAS, Note on the flux problem for stationary Navier-Stokes equations in domains with multiply connected boundary, Acta App. Math. 37 (1994), 21-30.

[3] J. Bourgain, M. V. Korob Kov and J. Kristensen, On the Morse-Sard property and level sets of Sobolev and BV functions, Rev. Mat. Iberoam. 29 (2013), 1-23.

[4] D. Burago, Yu. Burago and S. Ivanov, "A Course in Metric Geometry", Graduate Studies in Mathematics, Vol. 33, AMS, 2001.

[5] R. R. Coifman, J. L. Lions, Y. Meier and S. Semmes, Compensated compactness and Hardy spaces, J. Math. Pures Appl. (IX) 72 (1993), 247-286.

[6] J. R. DorRonsoro, Differentiability properties of functions with bounded variation, Indiana Univ. Math. J. 38 (1989), 1027-1045.

[7] L. C. Evans And R. F. Gariepy, "Measure Theory and fine Properties of Functions", Studies in Advanced Mathematics, CRC Press, Boca Raton, FL, 1992. 
[8] R. FInN, On the steady-state solutions of the Navier-Stokes equations. III, Acta Math. 105 (1961), 197-244.

[9] H. FuJita, On the existence and regularity of the steady-state solutions of the NavierStokes theorem, J. Fac. Sci. Univ. Tokyo Sect. I 9 (1961), 59-102.

[10] H. FUJITA, On stationary solutions to Navier-Stokes equation in symmetric plane domain under general outflow condition, In: "Proceedings of International conference on NavierStokes Equations. Theory and Numerical Methods", June 1997, Varenna, Italy, Pitman Research Notes in Mathematics, Vol. 388, (1997), 16-30.

[11] G. P. GALDI, On the existence of steady motions of a viscous flow with non-homogeneous conditions, Matematiche (Catania) 66 (1991), 503-524.

[12] G. P. GALDI, "An Introduction to the Mathematical Theory of the Navier- Stokes Equation. Steady-state Problems", second edition, Springer, 2011.

[13] L. V. KAPITANSKII and K. PILECKAS, On spaces of solenoidal vector fields and boundary value problems for the Navier-Stokes equations in domains with noncompact boundaries, Trudy Mat. Inst. Steklov 159 (1983), 5-36. English Transl., Proc. Math. Inst. Steklov 159 (1984), 3-34.

[14] M. V. Korob Kov, Bernoulli law under minimal smoothness assumptions, Dokl. Math. 83 (2011), 107-110.

[15] M. V. Korobkov, K. Pileckas and R. Russo, On the flux problem in the theory of steady Navier-Stokes equations with nonhomogeneous boundary conditions, Arch. Ration. Mech. Anal. 207 (2013), 185-213.

[16] M. V. Korob Kov, K. PILECKAS and R. Russo, Steady Navier-Stokes system with nonhomogeneous boundary conditions in the axially symmetric case, Comptes Rendus Mécanique 340 (2012), 115-119.

[17] M. V. KorobKov, K. Pileckas and R. Russo, Solution of Leray's problem for stationary Navier-Stokes equations in plane and axially symmetric spatial domains, Ann. of Math. 181 (2015), 769-807.

[18] H. KozONO and T. YANAGISAWA, Leray's problem on the stationary Navier-Stokes equations with inhomogeneous boundary data, Math. Z. 262 (2009), 27-39.

[19] A. S. KRONROD, On functions of two variables, Uspekhi Mat. Nauk (N.S.) 5 (1950), 24134 (in Russian).

[20] O. A. LADYZHENSKAYA, Investigation of the Navier-Stokes equations in the case of stationary motion of an incompressible fluid, Uspekhi Mat. Nauk 3 (1959), 75-97 (in Russian).

[21] O. A. LAdyzhenskaya, "The Mathematical Theory of Viscous Incompressible Fluid", Gordon and Breach, 1969.

[22] O. A. LADYZHENSKAYA and V. A. SolonNiKov, On some problems of vector analysis and generalized formulations of boundary value problems for the Navier-Stokes equations, Zapiski Nauchn. Sem. LOMI 59 (1976), 81-116 (in Russian); English translation in Journal of Soviet Mathematics 10 (1978), 257-286.

[23] J. LERAY, Étude de diverses équations intégrales non linéaire et de quelques problèmes que pose l'hydrodynamique, J. Math. Pures Appl. 12 (1933), 1-82.

[24] V. G. MAZ’YA, “Sobolev Spaces”, Springer-Verlag, 1985.

[25] C. Miranda, "Partial Differential Equations of Elliptic Type", Springer-Verlag, 1970.

[26] H. Morimoto, A remark on the existence of 2-D steady Navier-Stokes flow in bounded symmetric domain under general outflow condition, J. Math. Fluid Mech. 9 (2007), 411418.

[27] J. NEUSTUPA, A new approach to the existence of weak solutions of the steady NavierStokes system with inhomoheneous boundary data in domains with noncompact boundaries, Arch. Ration. Mech. Anal. 198 (2010), 331-348.

[28] V. V. PUKHNACHEV, Viscous Flows in domains with a multiply connected boundary, In: "New Directions in Mathematical Fluid Mechanics", The Alexander V. Kazhikhov Memorial Volume, Fursikov A. V., Galdi G. P. and Pukhnachev V. V. (eds.), Basel-Boston-Berlin: Birkhäuser, 2009, 333-348. 
[29] V. V. Pukhnachev, The Leray problem and the Yudovich hypothesis, Izv. vuzov. Sev.Kavk. region. Natural sciences. Special issue "Actual problems of mathematical hydrodynamics" (2009), 185-194 (in Russian).

[30] G. V. AleKSEEV and V. V. PUKHnACheV, The axially symmetric flow through problem for the Navier-Stokes equations in variables "Vorticity-Stream Function", Dokl. Phys. 57 (2012), 301-306.

[31] A. RUSSO, A note on the two-dimensional steady-state Navier-Stokes problem, J. Math. Fluid Mech. 11 (2009), 407-414.

[32] R. Russo, On the existence of solutions to the stationary Navier-Stokes equations, Ricerche Mat. 52 (2003), 285-348.

[33] V. A. SOLONNIKOV and V. E. SCADILOV, On a boundary value problem for a stationary system of Navier-Stokes equations, Proc. Steklov Inst. Math. 125 (1973), 186-199 (in Russian).

[34] E. STEIn,"Harmonic Analysis: Real-Variables Methods, Orthogonality and Oscillatory Integrals", Princeton University Press, 1993.

[35] A. TAKeshitA, A remark on Leray's inequality, Pacific J. Math. 157 (1993), 151-158.

[36] I. I. Vorovich AND V. I. YUdOVICH, Stationary flows of a viscous incompressible fluid, Mat. Sbornik 53 (1961), 393-428 (in Russian).

Sobolev Institute of Mathematics

Koptyuga pr. 4

and Novosibirsk State University

Pirogova Str. 2

630090 Novosibirsk, Russia

korob@math.nsc.ru

Faculty of Mathematics and Informatics

Vilnius University

Naugarduko Str., 24

Vilnius, 03225 Lithuania

pileckas@ktl.mii.lt

Dipartimento di Matematica e Fisica

Seconda Università di Napoli

Via Vivaldi, 43

81100 Caserta, Italia

remigio.russo@unina2.it 